\title{
GPx3 dysregulation impacts adipose tissue insulin receptor expression and sensitivity
}

\author{
Robert Hauffe, ${ }^{1,2}$ Vanessa Stein,, ${ }^{1,2}$ Chantal Chudoba, ${ }^{1,2}$ Tanina Flore, ${ }^{1,2}$ Michaela Rath, ${ }^{1,2}$ \\ Katrin Ritter, ${ }^{1,2}$ Mareike Schell, ${ }^{1,2}$ Kristina Wardelmann, ${ }^{1,2}$ Stefanie Deubel, ${ }^{3}$ \\ Johannes Florian Kopp, ${ }^{4,5}$ Maria Schwarz,, 5 Kai Kappert, ${ }^{7}$ Matthias Blüher, ${ }^{8}$ Tanja Schwerdtle, ${ }^{4,5}$ \\ Anna P. Kipp, ${ }^{5,6}$ and André Kleinridders ${ }^{1,2,9}$ \\ IJunior Research Group Central Regulation of Metabolism, German Institute of Human Nutrition, Nuthetal, Germany. \\ ${ }^{2}$ German Center for Diabetes Research (DZD), München-Neuherberg, Germany. ${ }^{3}$ Department of Molecular Toxicology, \\ German Institute of Human Nutrition, Nuthetal, Germany. ${ }^{4}$ Institute of Nutritional Science, Department of Food Chemistry, \\ University of Potsdam, Nuthetal, Germany. ${ }^{5}$ DFG-Research Group \#2558 TraceAGE Potsdam-Berlin-Jena, Germany. \\ ${ }^{6}$ Institute of Nutritional Sciences, Department of Molecular Nutritional Physiology, Friedrich Schiller University Jena, Jena, \\ Germany. ${ }^{7}$ Institute of Laboratory Medicine, Clinical Chemistry and Pathobiochemistry, Charité - Universitätsmedizin \\ Berlin, corporate member of Freie Universität Berlin, Humboldt-Universität zu Berlin, and Berlin Institute of Health, Berlin, \\ Germany. ${ }^{8}$ Department of Medicine, University of Leipzig, Leipzig, Germany. ${ }^{9}$ Institute of Nutritional Science, Department of \\ Molecular and Experimental Nutritional Medicine, University of Potsdam, Nuthetal, Germany.
}

Insulin receptor signaling is crucial for white adipose tissue (WAT) function. Consequently, lack of insulin receptor (IR) in WAT results in a diabetes-like phenotype. Yet, causes for IR downregulation in WAT of patients with diabetes are not well understood. By using multiple mouse models of obesity and insulin resistance, we identify a common downregulation of IR with a reduction of mRNA expression of selenoproteins Txnrd3, Sephs2, and Gpx3 in gonadal adipose tissue. Consistently, GPX3 is also decreased in adipose tissue of insulin-resistant and obese patients. Inducing Gpx3 expression via selenite treatment enhances IR expression via activation of the transcription factor Sp1 in 3T3-L1 preadipocytes and improves adipocyte differentiation and function. Feeding mice a selenium-enriched high-fat diet alleviates diet-induced insulin resistance with increased insulin sensitivity, decreased tissue inflammation, and elevated IR expression in WAT. Again, IR expression correlated positively with Gpx3 expression, a phenotype that is also conserved in humans. Consequently, decreasing GPx3 using siRNA technique reduced IR expression and insulin sensitivity in 3T3-L1 preadipocytes. Overall, our data identify GPX3 as a potentially novel regulator of IR expression and insulin sensitivity in adipose tissue.

Conflict of interest: The authors have declared that no conflict of interest exists.

Copyright: (ㄷ) 2020, American Society for Clinical Investigation.

Submitted: January 10, 2020

Accepted: April 29, 2020

Published: May 5, 2020.

Reference information: /CI Insight. 2020;5(11):e136283.

https://doi.org/10.1172/jci.

insight.136283.

\section{Introduction}

The steadily increasing occurrence of obesity with concomitant insulin resistance and adipocyte dysfunction constitutes a serious health problem worldwide. A common feature is the presence of low-grade inflammation and oxidative stress in tissues of obese, insulin-resistant animals and patients (1). In particular, low-grade inflammation represents a major contributor to the establishment of insulin resistance in adipose tissue and has been intensively investigated in the last decades (2). Moreover, excessive oxidative stress can cause insulin resistance and is linked to inflammation in adipocytes, whereas low amounts of hydrogen peroxide exhibit insulin-mimetic effects (3). Thus, a fine-balanced redox status is needed to obtain optimal insulin sensitivity in adipose tissue (4).

More than 40 years ago, it was observed that insulin receptor (IR) expression is downregulated in obese humans and diabetic patients $(5,6)$. This downregulation is also present in adipocytes of patients with diabetes and insulin-resistant animals $(7,8)$. Lack of insulin signaling causes adipocyte dysfunction, linking obesity, insulin resistance, and diabetes (9). Indeed, loss of IR expression in adipose tissue results in both diabetes and a marked reduction in white adipose tissue mass (10). These data indicate that counteracting the decline in IR expression and finely balancing oxidants during obesity development can improve metabolism. 
The trace element selenium exerts its biological functions via selenoproteins and plays a crucial role in cellular redox balance, immunity, and metabolism. Yet, loss of individual selenoproteins in mice can result in a variety of outcomes with opposing results on metabolic health. Thus, whole-body deletion of SelenoP or glutathione peroxidase (GPx) 4 in mice both lead to severe neurological defects. However, whereas a SelenoM-deficient mouse model is susceptible to the development of obesity, GPx1 deletion alleviates insulin resistance induced by high-fat diet (HFD) (11-14). These results reflect the importance of a selenium supply that maintains the expression of important selenoproteins for human health. Most European populations exhibit a suboptimal selenium status below recommended values (15), with some studies revealing even poorer selenium status in obese children and obese females $(16,17)$. However, high-selenium diet can also induce insulin resistance (18), and high levels of selenium intake might increase the risk for diabetes (19), indicating that selenium intake and selenium-dependent processes must be tightly regulated to propagate insulin sensitivity and healthy metabolism.

Interestingly, selenium in the form of selenate exerts insulin-like effects, such as upregulation of glucose transporter or enhancing insulin-induced activation of IR in adipocytes (20). Further, GPx3 is a marker of differentiated adipocytes $(21,22)$, suggesting that selenoproteins are crucial for proper adipose tissue function. Although GPx3 is considered a plasma glutathione peroxidase, which is predominantly expressed by the kidney, it has been shown that GPx3 is also expressed in adipocytes, represents an adipokine in humans, and belongs to a cluster related to insulin sensitivity $(22,23)$.

So far it is not well understood why IR expression decreases in adipose tissue in diabetes and whether this reduction correlates with altered selenium supply. It has been shown that in vitro, insulin treatment per se can reduce IR expression (24), and nuclear FoxO1 is able to increase IR expression (25). Yet, hyperinsulinemia, a feature of insulin-resistant states, causes selective insulin resistance in adipocytes where insulin-induced FoxO1 nuclear exclusion remains preserved (26), indicating that altered FoxO1 regulation might not fully explain reduced expression of the IR in diabetic conditions.

In this study, we show that a combination of hyperglycemia, long-term insulin resistance, and obesity, but not short-term insulin resistance, in a variety of animal models, is linked to reduced IR and selenoprotein Gpx3, selenophosphate synthetase 2 (Sephs2), and thioredoxin reductase 3 (Txnrd3) mRNA expression in adipose tissue. Strikingly, the reduction of GPX3 was also confirmed in overweight/obese patients, which has been previously shown for obese and insulin-resistant patients (27). On a cellular level, we show that inducing selenoprotein expression in 3T3-L1 preadipocytes increases IR levels, resulting in enhanced insulin-induced glucose uptake and adipocyte differentiation. This appears to be mediated by activation of the transcription factor Sp1 because Sp1 inhibition reduces selenite-induced IR regulation and reduces adipocyte differentiation. In vivo, selenite supplementation improves insulin sensitivity in HFD-fed animals with elevated IR expression and signaling in gonadal adipose tissue. We identify a positive correlation of $I r$ expression with the expression of selenoproteins Gpx3, Sephs2, and Txnrd3 in white adipose tissue in animals. Strikingly, the correlation of $I R$ and GPX3 mRNA expression was conserved in humans. We further show that Gpx3 is crucial for selenite-induced IR expression and signaling in white adipocytes. Taken together, we describe a potentially novel, functional interaction of the selenotranscriptome and insulin action in adipose tissue, identifying GPx 3 as a novel regulator of IR in white adipocytes.

\section{Results}

To determine whether obesity, altered leptin, or insulin caused a reduction of IR expression in adipose tissue, we investigated IR expression in gonadal white adipose tissue (gWAT) of obese (ob/ob, 12 weeks of age), diabetic ( $\mathrm{db} / \mathrm{db}, 12$ weeks of age), and ovariectomy-induced insulin-resistant mice (16 weeks of age) and C57BL/6N mice fed an HFD for 3 days or 12 weeks. This analysis revealed that obesity and lack of leptin signaling are not sufficient to cause a reduction in Ir expression in gWAT because Ir mRNA levels were unchanged between control (ob/+) and normoglycemic obese, leptin-deficient (ob/ob) mice (Figure 1, A and C; and Supplemental Figure 1A; supplemental material available online with this article; https:// doi.org/10.1172/jci.insight.136283DS1). Yet obese, diabetic db/db mice exhibited an approximately 30\% reduction in gonadal Ir expression, suggesting that combined insulin and leptin resistance with obesity is necessary to decrease Ir expression (Figure 1A and Supplemental Figure 1B). Thus, we investigated whether short-term HFD feeding in male C57BL/6N mice, which induces insulin resistance, was able to reduce Ir expression in gWAT. Three days of HFD caused insulin resistance and hyperglycemia (for metabolic data see ref. 28) but did not affect body weight. Ir expression in gWAT showed only a slight trend 
to be decreased, indicating that short-term insulin resistance per se is not sufficient to reduce Ir expression in adipose tissue (Figure 1B). Following, we fed mice an HFD for 12 weeks, which caused hyperglycemia, insulin resistance, and obesity (Supplemental Figure 1C). Here, hyperglycemia, long-term insulin resistance (LTIR), and obesity resulted in significantly decreased IR expression in gWAT, which was also evident for $\mathrm{db} / \mathrm{db}$ and ob/ob mice (Figure 1, B and C; and Supplemental Figure 1E). These data suggest that the combination of hyperglycemia, LTIR, and obesity (HIRO) is a determining factor that contributes to reduced gene and protein expression of IR. To further investigate this hypothesis, we examined Ir expression in ovariectomized female mice fed an HFD as an alternative model of insulin resistance with obesity. Ovariectomy exhibited a strong tendency to increased blood glucose levels, increased body weight, caused insulin resistance, and led to a reduction of Ir expression in gWAT compared with sham-operated controls (Figure 1, D-F; and Supplemental Figure 1, D and F).

Reduced insulin action in adipose tissue is also linked to elevated oxidative stress, the latter being modulated by different selenoproteins. Thus, we investigated the gWAT selenotranscriptome, consisting of 25 selenoproteins, in the aforementioned mouse models. This analysis revealed that all investigated metabolically impaired mouse models exhibited unique differences compared with their healthy control groups with the exception of a common reduction of gene expression of Gpx3, Sephs2, and Txnrd3 (Figure 1, G-I), which was confirmed on protein level for GPx3, with unexpectedly reduced protein expression of GPx3 in ob/ob mice also (Figure 1J and Supplemental Figure 1, E and F). Interestingly, Gpx3, Sephs2, and Txnrd3 were uniformly reduced in gWAT of HIRO mouse models compared with healthy controls (Figure $1 \mathrm{~K}$ and Supplemental Figure 1G). A reduction of GPX3 was also observed when comparing adipose tissue of overweight and obese and normal-weight patients (Figure 1L and clinical parameters in Supplemental Table 2). SEPHS2 and TXNRD3 expression levels were unaltered between lean and obese patients (Supplemental Figure $1 \mathrm{H})$. Interestingly, insulin-like growth factor receptor (Igflr) expression was unaltered in our HIRO mouse models, indicating a specific IR regulation (Supplemental Figure 1I).

Because supplementation with selenite can dose-dependently modulate selenoprotein expression as well as impact cellular function, we aimed to identify an optimal treatment concentration. Thus, we incubated 3T3-L1 preadipocytes with $200 \mathrm{nM}, 500 \mathrm{nM}, 1 \mu \mathrm{M}, 5 \mu \mathrm{M}$, and $10 \mu \mathrm{M}$ selenite for 96 hours to allow for complete selenoprotein turnover. This analysis identified the range between 200 and $1000 \mathrm{nM}$ selenite concentrations as ineffective with respect to reduction of cell viability, as assessed by lactate dehydrogenase $(\mathrm{LDH})$ activity and lactate concentrations in the supernatant (Supplemental Figure 2, A and B). Because $200 \mathrm{nM}$ is a commonly found concentration for in vitro studies, did not elicit negative effects on cellular homeostasis but induced $G p x 1$ and $G p x 3$ expression (as a marker of selenium incorporation via selenoprotein synthesis), and was shown to be optimal for GPx3 regulation (29), we used $200 \mathrm{nM}$ selenite in our conducted study (Supplemental Figure 2, C and D).

Next, we investigated whether selenite supplementation was able to alter Gpx3, Sephs2, or Ir expression in 3T3-L1 preadipocytes. We did not further investigate Txnrd3 because its expression levels in 3T3-L1 preadipocytes were very low and close to the detection limit. Selenite caused increased mRNA levels of $I r$, which were at earliest detectable after 48 hours of selenite supplementation, with a robust regulation after 96 hours, suggesting that alterations of different selenoproteins are involved in inducing Ir expression (Figure 2A). This increase was also present on protein levels and accompanied by increased Gpx3 gene expression, showing that selenite treatment successfully stimulated IR and Gpx3 expression (Figure 2, B and C). Igflr expression was unaltered in selenite-treated cells, pointing to IR specificity (Supplemental Figure 1I). Unexpectedly, Sephs 2 mRNA expression was downregulated after 96 hours of selenite treatment (Supplemental Figure 2E). To further study the effect of selenite treatment on insulin action, untreated and selenite-treated 3T3-L1 cells were stimulated with $100 \mathrm{nM}$ insulin for 5 minutes. This analysis revealed enhanced insulin signaling in selenite-treated cells as evidenced by elevated $\mathrm{AKT}_{\text {Ser473 }}$ phosphorylation with a concomitant $54 \%$ increase in IR expression (Figure 2D). Increased GPx1 protein expression confirmed successful selenite supplementation (Figure 2D). Because insulin action is crucial for adipocyte development, we investigated the effect of selenite treatment on adipocyte differentiation. Selenite treatment enhanced adipocyte differentiation compared with control with a 70\% increase in Oil Red O staining and concurrent increases in Pparg, Glut4, and Ap2 mRNA expression as well as increased Ir mRNA levels. Increased Gpx1 and SelenoP mRNA levels showed successful selenite stimulation (Figure 2, E and F). Interestingly, both Glut4 and Ir were already upregulated in selenite-treated cells before differentiation, suggesting that selenite increases susceptibility to differentiation of 3T3-L1 cells (Figure 2G). In differentiated 

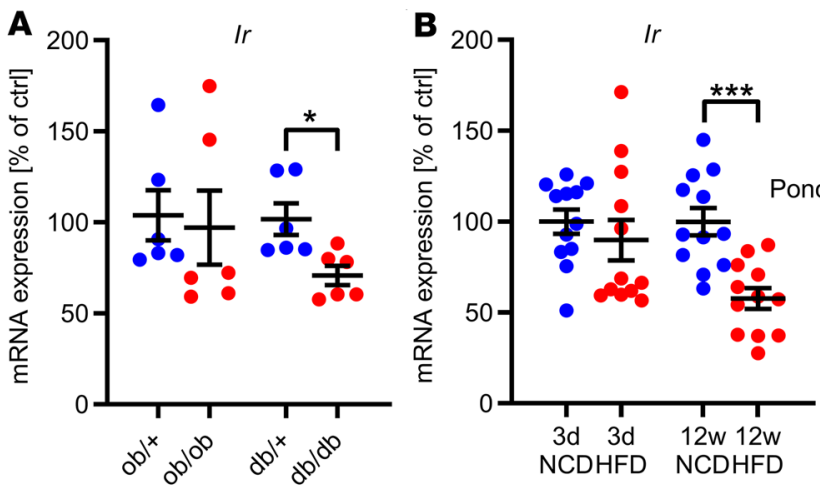

C
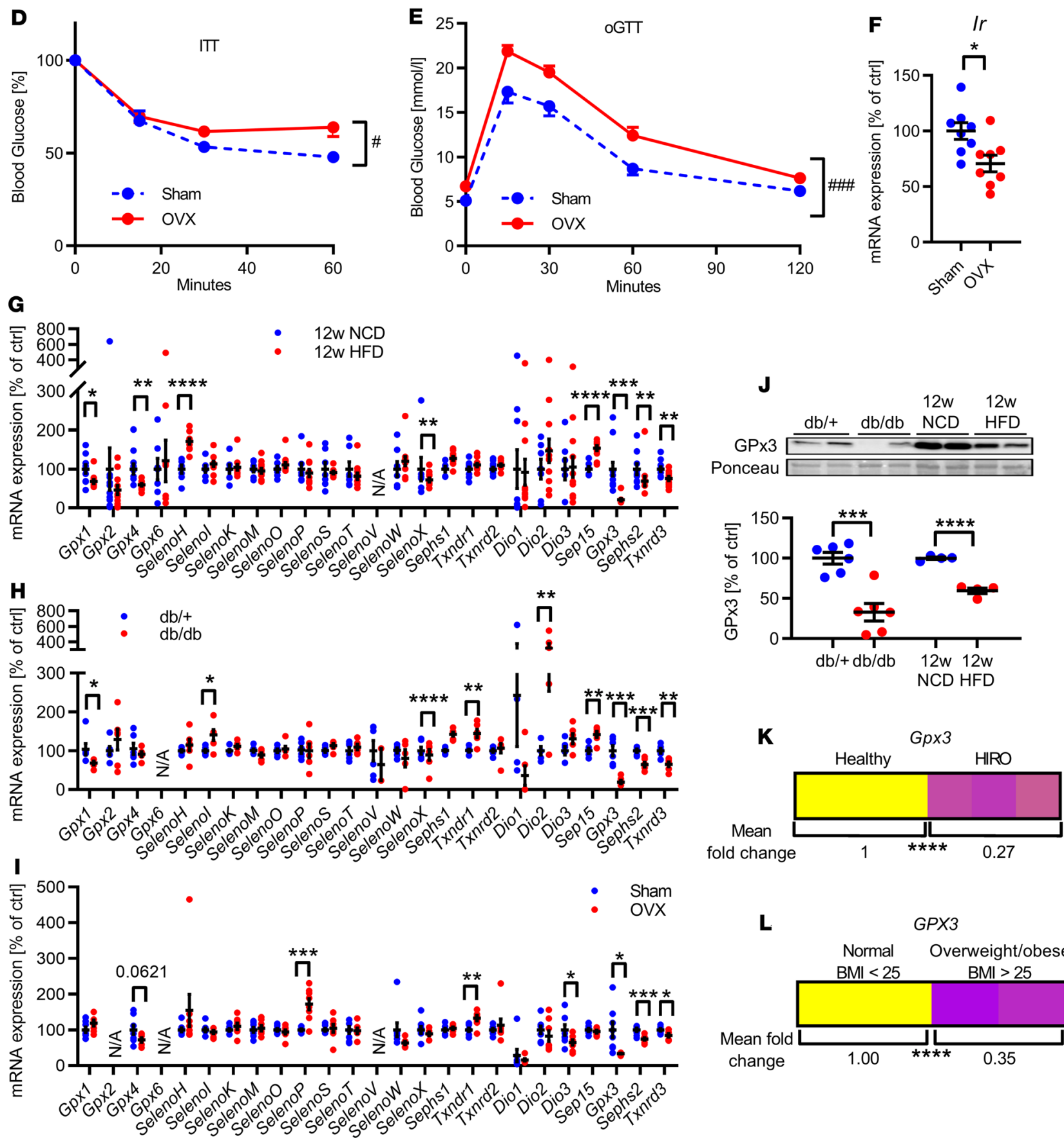

$\mathbf{L}$

GPX3

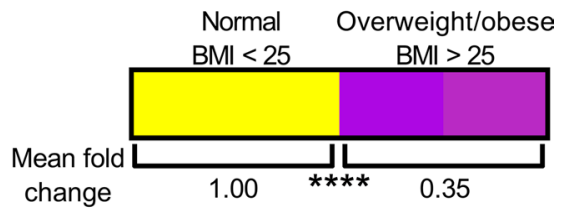


Figure 1. Long-term insulin resistance causes downregulation of IR in epigonadal adipose tissue and associates with altered selenotranscriptome. (A) Gonadal adipose tissue (gWAT) expression of $I r$ mRNA in genetic models of obesity at 12 weeks of age $(n=6)$. (B) gWAT expression of $I r$ in male C57BL/6N mice fed normal chow diet (NCD) or HFD for 3 days or 12 weeks $(n=11-12)$. (C) Representative gWAT IR expression in aforementioned mouse models (densitometry $n=4$ ). ( $\mathbf{D}$ and $\mathbf{E}$ ) Blood glucose levels during insulin tolerance test (ITT) (D) or oral glucose tolerance test (oGTT) (E) of female sham or ovariectomized (OVX) mice fed HFD for 14 weeks $(n=12)$. (F) gWAT expression of $I r$ in sham or OVX mice fed HFD $(n=8)$. (G-I) gWAT mRNA expression of the selenoprotein transcriptome in aforementioned mouse models $(n=6-12)$. (J) Representative gWAT GPx3 protein expression in male mouse models of HIRO (densitometry $n=4$ ). ( $\mathbf{K}$ and $\mathbf{L}$ ) Combined mean fold change of Gpx3 mRNA expression in gWAT of healthy and HIRO (K, $n=6-12)$ and of WAT of normal-weight (BMI $<25, n=88$ ) and overweight/obese (BMI $>25, n=274)$ human patients $(\mathrm{L})$. ${ }^{*} P<0.05$, ${ }^{* *} P<0.01,{ }^{* * *} P<0.001$, and ${ }^{* * * *} P<0.0001$ after 2 -tailed Student's $t$ test. ${ }^{\#} P<0.05$, and ${ }^{\# \#} P<0.001$ after 2 -way ANOVA. All data are presented as mean \pm SEM.

adipocytes, selenite treatment also increased glucose uptake under basal and insulin-stimulated conditions, confirming beneficial effects of selenite treatment on adipocyte function (Figure $2 \mathrm{H}$ ). These data show that selenite treatment enhances adipocyte function by modulating IR expression. The transcription factor Sp1 has been shown to regulate Ir gene expression (30). To test whether selenite treatment enhances $I r$ expression and adipocyte function via Sp1, we costimulated untreated and selenite-treated preadipocytes with the Sp1 inhibitor mithramycin. Mithramycin attenuated the selenite-induced effect on Ir expression, while not changing basal Ir levels (Figure 2I). In line, mithramycin reduced adipocyte differentiation compared with control, as evidenced by reduced Oil Red O staining (Figure 2J). Of note, mithramycin reduced SpI expression but did not generally decrease cellular mRNA synthesis or induce cellular stress, as evidenced by unchanged expression levels of endoplasmatic stress markers $A t f 4$ and Chop, as well as mitochondrial stress markers Hsp60, Hsp10, LonP, and ClpP (Supplemental Figure 2F).

Next, we characterized the effect of selenite treatment on mitochondrial function because mitochondria are essential for proper adipocyte function and adipose tissue development (31). Selenite treatment increased basal respiration by 2-fold in 3T3-L1 preadipocytes while not altering oxidative stress levels, as evidenced by indistinguishable protein carbonylation between groups (Figure 3, A and B). This selenite-induced increase in mitochondrial function could not be explained by improved mitochondrial proteostasis because overall mRNA levels of members of the mitochondrial unfolded protein response were unchanged and protein expression of subunits of the electron transport chain complexes were unaltered (Figure 3, C and D). There was no difference in mitochondrial DNA copy number (Figure 3E). In addition, selenite treatment resulted in decreased mRNA levels of master regulators of mitochondrial biogenesis Pgcla and mitochondrial transcription factor Tfam, leading us to question whether the selenite-induced increase in mitochondrial function was caused by altered biogenesis (Figure 3F). Because Glut4 expression was increased 2-fold in selenite-treated compared with control cells (Figure $2 \mathrm{G}$ ), increased glucose availability might be responsible for higher basal respiration rates. To investigate this hypothesis, we permeabilized 3T3-L1 preadipocytes, allowing unregulated metabolite flux to mitochondria. Permeabilizing 3T3-L1 cells with saponin and providing pyruvate and malate as substrates showed no differences in basal respiration between untreated and selenite-treated cells (Figure 3G). Thus, increased substrate availability due to elevated Glut4 expression seems to be responsible for the elevated mitochondrial respiration.

Next, we investigated whether supplementing an HFD with selenite was able to improve metabolism by counteracting the decline of IR expression in gWAT during obesity development. We fed C57BL/6N mice of both sexes a conventional HFD containing $230 \mathrm{ng}$ selenite/g diet, which is above the recommendation for mice of $150 \mathrm{ng} / \mathrm{g}$, and a selenium-rich HFD (SRHFD) containing $670 \mathrm{ng}$ selenite/g diet for 12 weeks (Supplemental Table 1). First, we assessed selenium concentrations in plasma, liver, brain, and gWAT of SRHFD-fed mice. We found an increase in plasma selenium levels in SRHFD-fed mice, with additionally elevated liver selenium content of $19 \%$ in females and $26 \%$ in males, respectively, whereas no changes were observed in brain tissues (Figure 4, A-C; and Supplemental Figure 3, A-C). Although gWAT was characterized by lowest selenium concentrations compared with other tested organs, ranging from $0.029 \mu \mathrm{g}$ to $0.048 \mu \mathrm{g} / \mathrm{g}$ tissue, it revealed highest selenite supplementation efficacy, as evidenced by a more than 2-fold increase in selenium content in the selenite-supplemented group (Figure 4D and Supplemental Figure 3D).

However, assessing the effect of selenite supplementation on energy homeostasis during obesity development revealed no alterations in body weight, food intake, blood glucose levels, energy expenditure, or respiratory exchange ratio (Figure 4, E-I; and Supplemental Figure 3, E-I). In addition, feeding mice an SRHFD showed no major impact on pancreas, skeletal muscle, or liver weight (Supplemental Figure 4). In line with increased selenium concentration in livers of mice fed an SRHFD (Figure 4B), liver GPx1 protein expression was increased by $25 \%$ in females and $72 \%$ in males, while total GPx activity was unaltered, suggesting that 
A

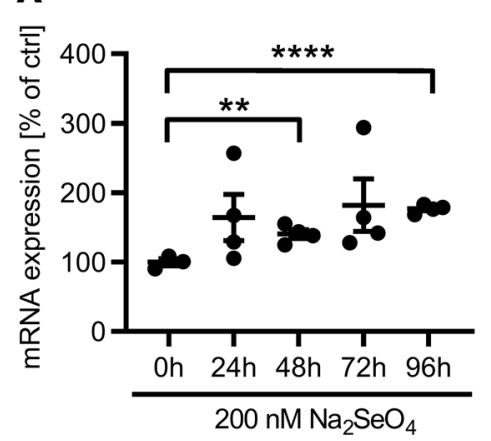

D

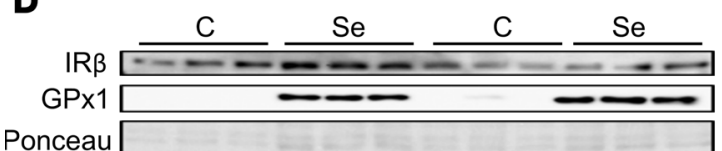

Ponceau

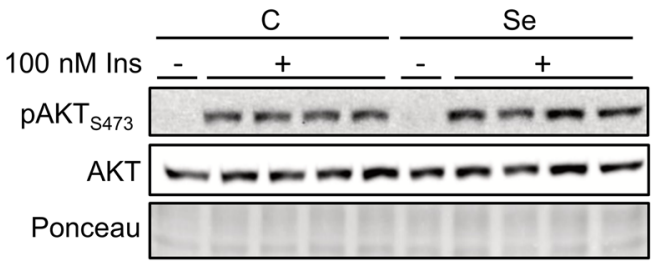

B

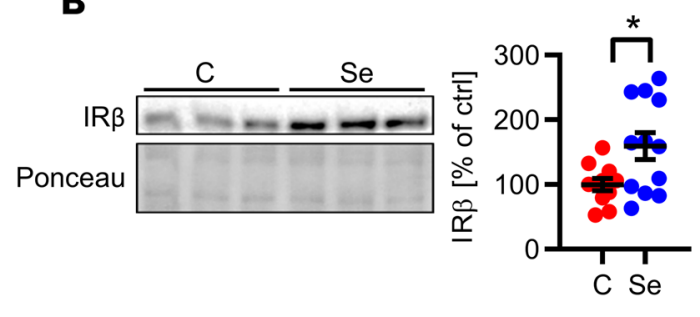

C

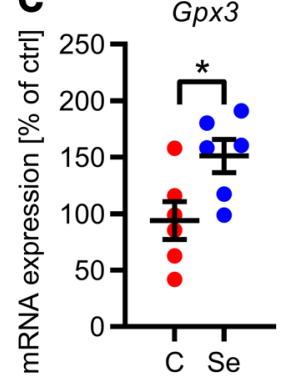

E
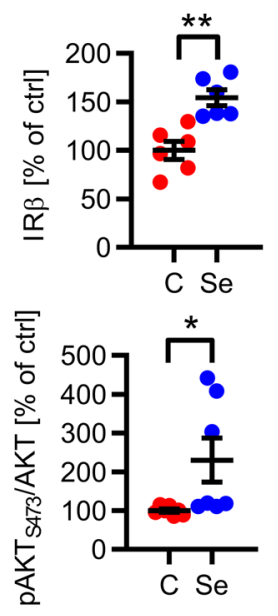

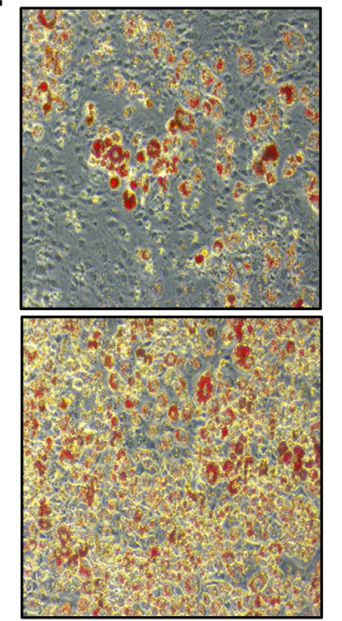

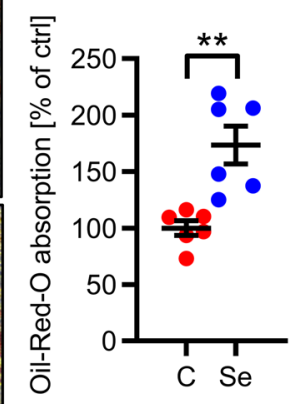

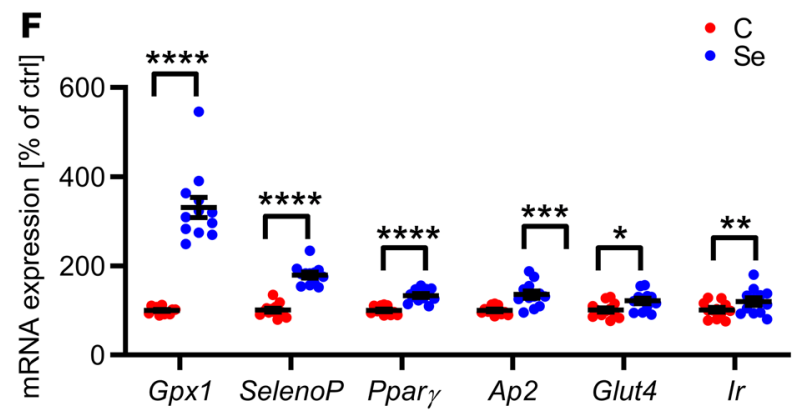

G
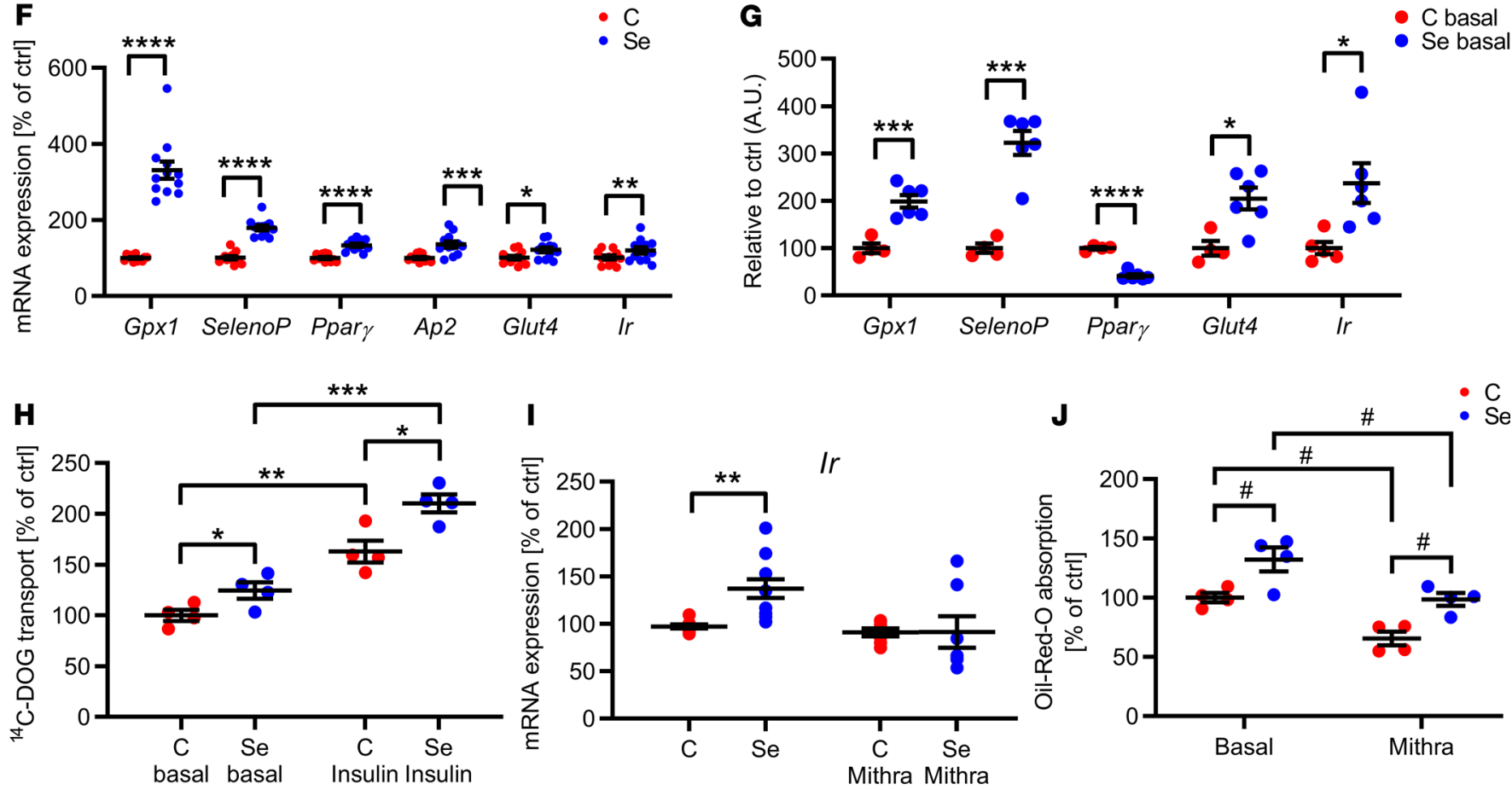

Figure 2. Selenite treatment increases IR expression and sensitivity in white adipocytes. (A) Ir expression in 3T3-L1 preadipocytes after selenite treatment. (B) Representative IR expression in 3T3-L1 preadipocytes after selenite treatment (densitometry $n=12$ ). (C) Gpx3 mRNA expression in 3T3-L1 preadipocytes after selenite treatment. (D) Western blot of members of the insulin signaling pathway and selenoprotein CPx1 after selenite and insulin treatment. (E) Representative microscopic image of Oil Red 0 staining and quantification of differentiated 3T3-L1 adipocytes after selenite treatment $(n=6)$. Original magnification, $\times 100$; side length, $500 \mu \mathrm{m}$. (F) Expression profile of adipocyte markers and selenoproteins of differentiated 3T3-L1 adipocytes after selenite treatment ( $n=12)$. (C) Expression profile of adipocyte markers and selenoproteins of undifferentiated 3T3-L1 preadipocytes after selenite treatment $(n=6)$. (H) Uptake of ${ }^{14} \mathrm{C}-$ labeled deoxy-D-glucose $\left({ }^{14} \mathrm{C}-\mathrm{DOC}\right)$ in differentiated 3T3-L1 adipocytes after selenite treatment $(n=4)$. (I) Ir mRNA expression in 3T3-L1 preadipocytes after selenite and 24 hours' mithramycin treatment; data were normalized to untreated control (condition C; $n=6$ ). (J) Oil Red O quantification of differentiated 3T3-L1 adipocytes after selenite and mithramycin treatment $(n=4) .{ }^{*} P<0.05,{ }^{* *} P<0.01$, ${ }^{* *} P<0.001$, and ${ }^{* * * *} P<0.0001$ after 2-tailed Student's $t$ test. ${ }^{*} P<0.05$ after 2-way ANOVA and Holm-Šídák post hoc test. All data are presented as mean \pm SEM. C, control; Se, 200-nM selenite treatment. 
A Basal respiration
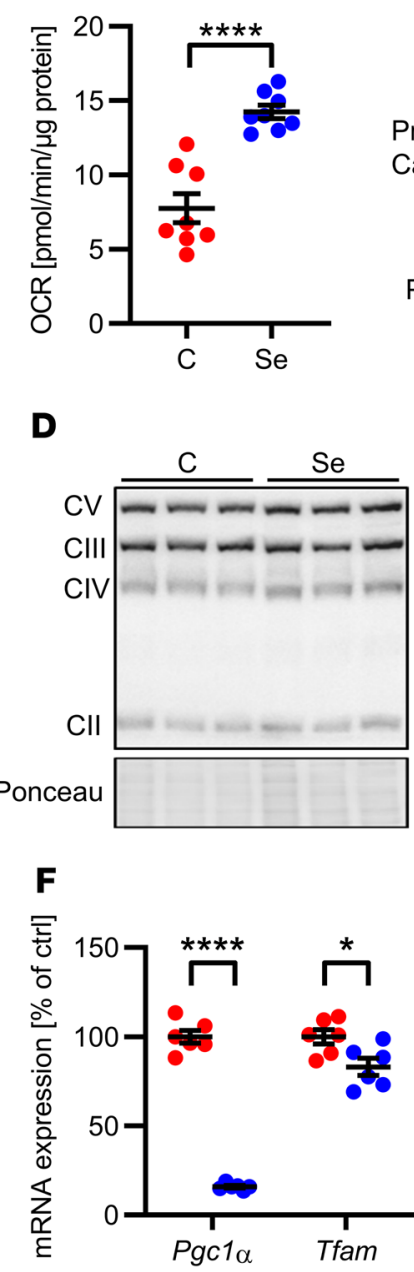

B

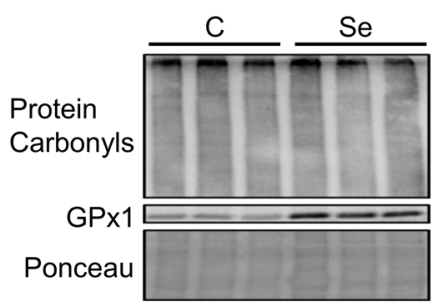

C

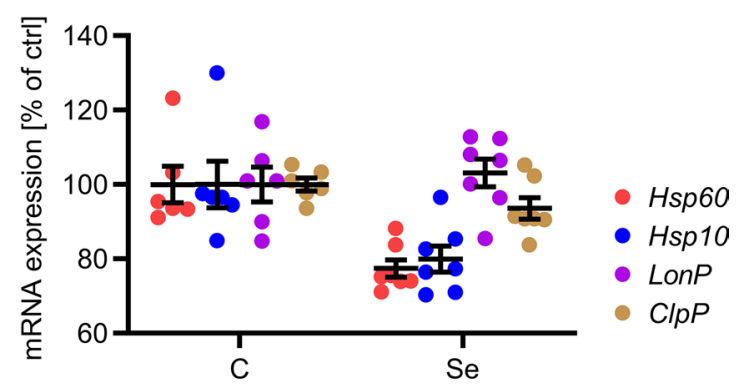

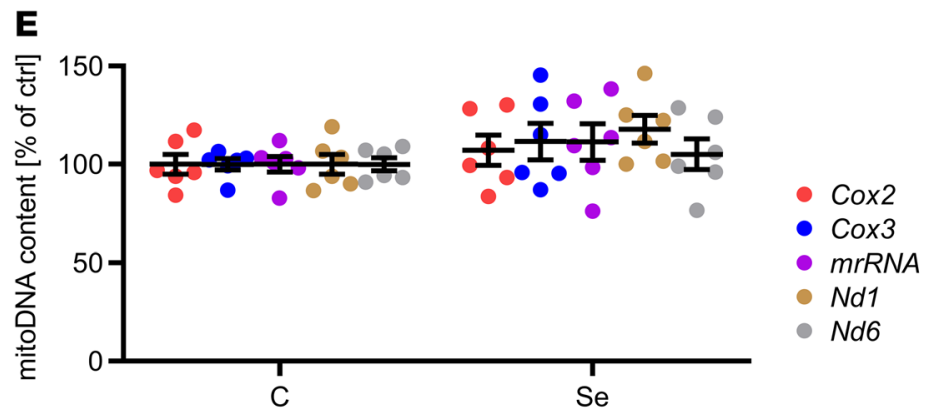

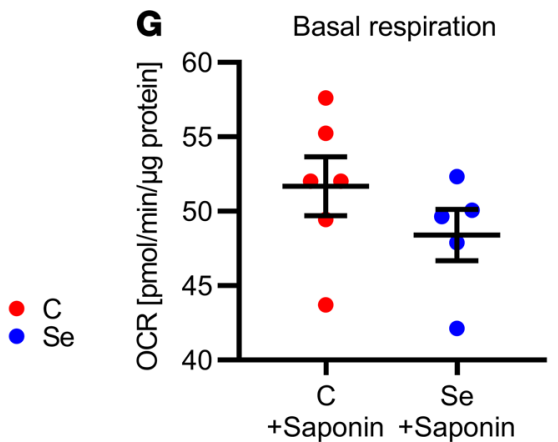

Figure 3. Selenite effect on mitochondrial function in white adipocytes. (A) Basal oxygen consumption rate (OCR) of 3T3-L1 preadipocytes after selenite treatment using the Seahorse Bioflux Analyzer XF96e $(n=8)$. (B) Protein carbonylation in 3T3-L1 preadipocytes after selenite treatment. (C) mRNA expression of members of the mitochondrial unfolded protein response in 3T3-L1 preadipocytes after selenite treatment $(n=6)$. (D) Protein expression of respiratory chain complexes in 3T3-L1 preadipocytes after selenite treatment. (E) Mitochondrial DNA content of 3T3-L1 preadipocytes after selenite treatment $(n=6)$. (F) mRNA expression of Pgc1a and Tfam in 3T3-L1 preadipocytes after selenite treatment $(n=6)$. (G) Basal oxygen consumption rate of 3T3-L1 preadipocytes after selenite treatment and permeabilization with saponin using the Seahorse Bioflux Analyzer XF96e $(n=7) .{ }^{*} P<0.05$, and ${ }^{* * * *} P$ $<0.0001$ after 2-tailed Student's $t$ test. All data are presented as mean \pm SEM.

GPx was already maximally activated under HFD conditions (Supplemental Figure 5, A-C). Although male mice fed an SRHFD exhibited a reduction in hepatic gene expression of several acyl-CoA dehydrogenases and increased Socs3 expression, only females exhibited reduced liver triglyceride $(P=0.0542)$ and nonesterified fatty acid (NEFA) levels with normal liver morphology (Supplemental Figure 5, D-G). In agreement with the unaltered selenium content in brains between control and SRHFD-fed mice, the hypothalamus exhibited no major differences in selenoprotein or ER stress-responsive gene expression levels, suggesting that the brain is protected against major alterations in selenium availability (Supplemental Figure 6).

In line with our hypothesis that selenite supplementation might influence adipose tissue biology, analysis of body composition using nuclear magnetic resonance (NMR) measurements revealed a 31\% reduction in adiposity assessed by NMR measurements in male mice fed an SRHFD. This was further supported by a $21 \%$ reduction in adipose tissue, calculated as the aggregate weight of dissected white adipose tissue depots of SRHFD- and HFD-fed mice relative to body weight (Figure 5, A and B). In contrast, females fed an SRHFD exhibited unaltered body composition (Supplemental Figure 7A). However, isolating different fat depots and calculating combined adipose tissue weight relative to body weight showed a $35 \%$ increase in combined adipose tissue weight compared with HFD control females (Supplemental Figure 7B). 
A

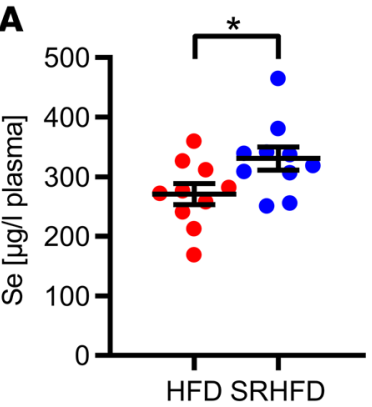

E

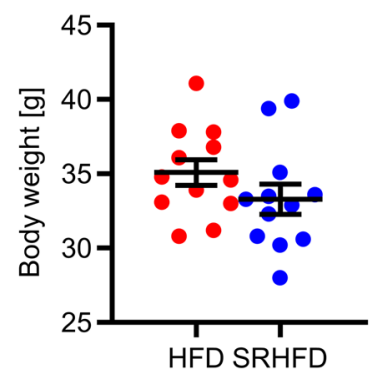

B

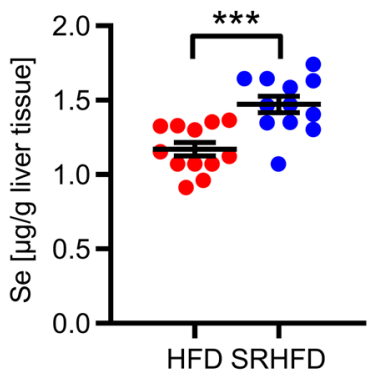

$\mathbf{F}$

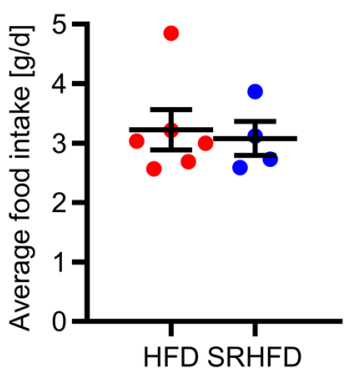

Resting

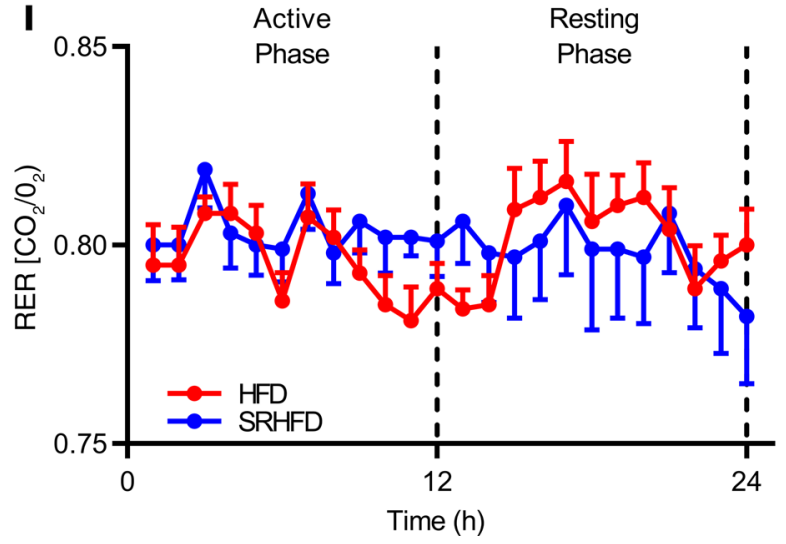

C

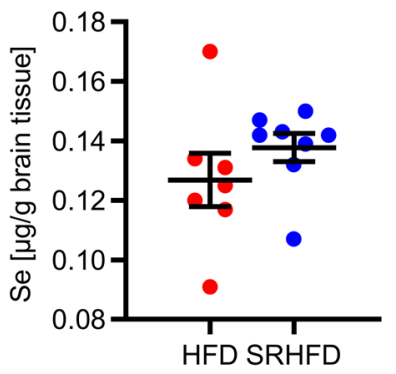

G

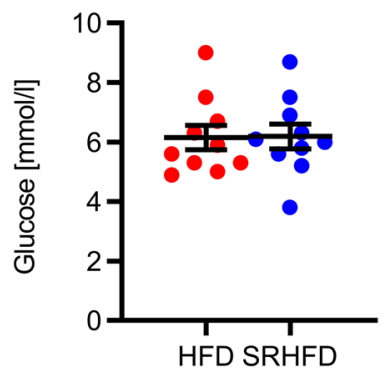

D

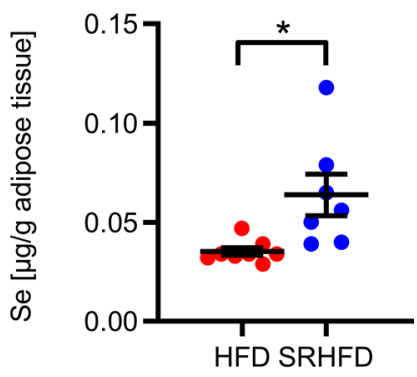

H

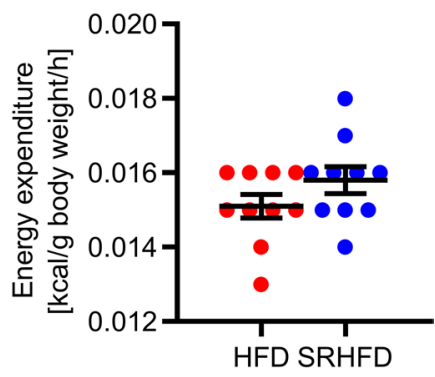

Figure 4. Unaltered energy homeostasis in male mice fed an SRHFD. (A-D) Selenium content in plasma (A), liver (B), brain (C), and adipose tissue (D) of mice after 12 weeks of either HFD or SRHFD $(n=8-12)$. (E) Body weight of mice after 12 weeks of either HFD or SRHFD $(n=8-12)$. (F) Average food intake over 24 hours of mice fed either HFD or SRHFD $(n=6)$. (C) Fasting blood glucose of mice fed either HFD or SRHFD $(n=8-12)$. (H) Energy expenditure in relation to body weight of mice fed either HFD or SRHFD $(n=12)$. (I) Respiratory exchange ratio of mice fed either HFD or SRHFD $(n=12) .{ }^{*} P<0.05$, and ${ }^{* * *} P<0.001$ after 2 -tailed Student's $t$ test. All data are presented as mean \pm SEM.

Although combined weight of all dissected adipose tissue depots was - though in opposite directions altered in both sexes, plasma leptin levels, triglycerides, and NEFAs were unchanged (Figure 5, C-E; and Supplemental Figure 7, C-E).

Interestingly, selenite supplementation increased mean adipocyte area in gWAT in both sexes (Figure $5 \mathrm{~F}$ and Supplemental Figure $7 \mathrm{~F}$ ). Although larger adipocytes tend to exhibit increased inflammatory markers (32), inflammation was reduced in gWAT of SRHFD-fed mice with reduced expression of F4/80, Tnfa, and $C c l 2$ and unaltered $I l 4$ expression (Figure 5G and Supplemental Figure 7G).

Reduced adipocyte inflammation is linked to improved metabolism. To further determine whether selenite supplementation affects glucose tolerance and insulin sensitivity during obesity development, we performed glucose and insulin tolerance tests in mice fed an HFD or SRHFD. Female and male mice fed an SRHFD exhibited comparable blood glucose levels during an oral glucose tolerance test compared with HFD control animals (Figure 6A and Supplemental Figure 7H). Strikingly, female and male mice fed an SRHFD exhibited improved insulin sensitivity compared with HFD control when challenged with a bolus injection of $0.75 \mathrm{U} / \mathrm{kg}$ insulin in an insulin tolerance test, showing an insulin-sensitizing effect of selenite supplementation and demonstrating that moderate selenite supplementation improves insulin 
A

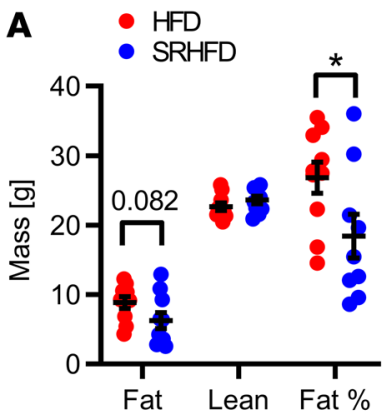

E

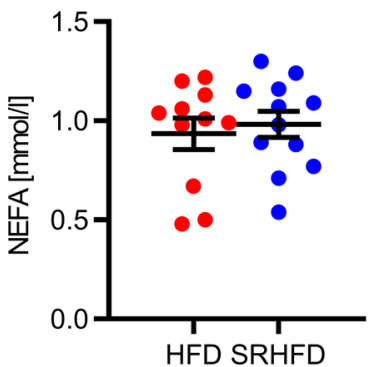

B

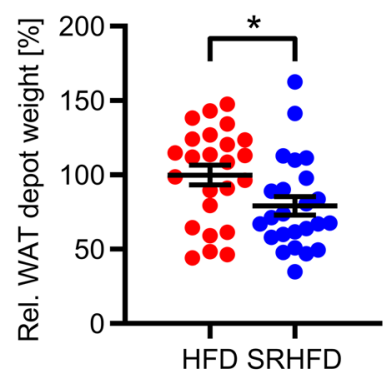

F

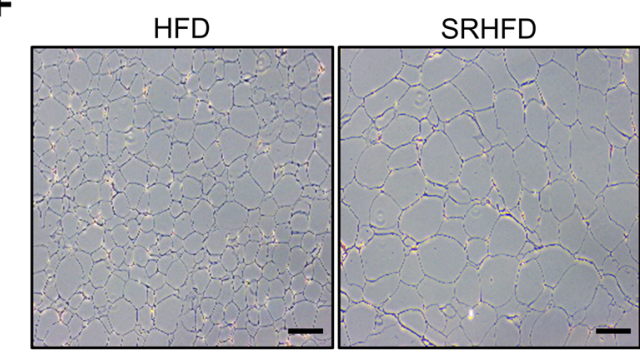

C

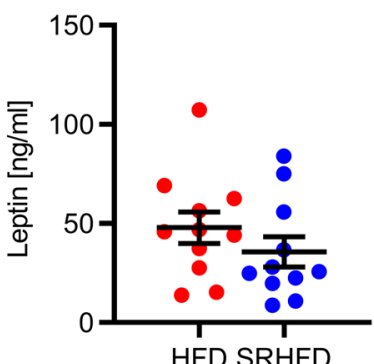

D

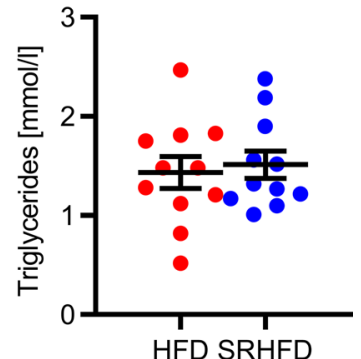

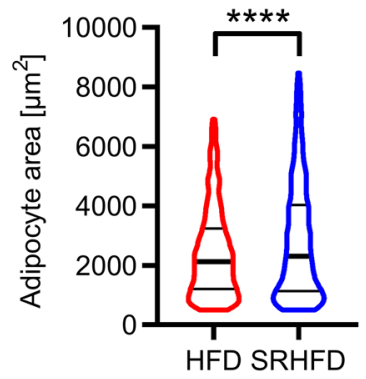

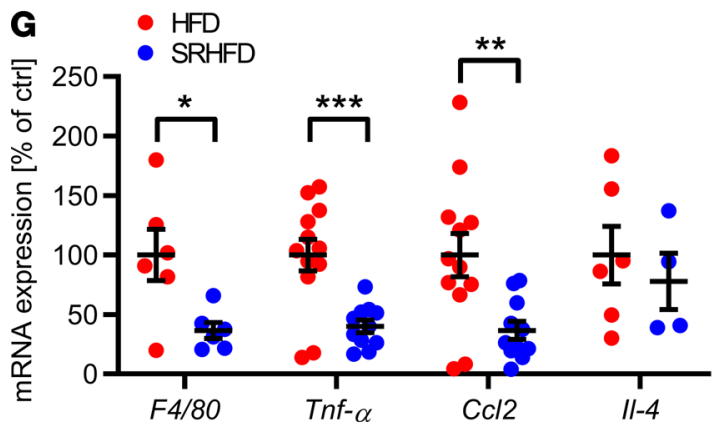

Figure 5. Selenite supplementation in diet-induced obesity alters adiposity in male mice. (A) Nuclear magnetic resonance (NMR) measurement of mice after 9 weeks of either HFD or SRHFD $(n=10)$. (B) Combined white adipose tissue (WAT) weight relative to body weight after 12 weeks of either HFD or SRHFD $(n=24)$. (C) Plasma leptin levels of mice after 12 weeks of either HFD or SRHFD $(n=12)$. (D and E) Plasma triglyceride (D) and nonesterified fatty acids (NEFAs, E) levels of mice after 12 weeks of either HFD or SRHFD $(n=12)$. (F) Representative hematoxylin and eosin stain and area analysis of gWAT of mice after 12 weeks of either HFD or SRHFD; scale bars: $100 \mu \mathrm{m} . n=6$. (G) mRNA expression levels of inflammatory markers in epigonadal adipose tissue of mice after 12 weeks of either HFD or SRHFD $(n=12) .{ }^{*} P<0.05,{ }^{* *} P<0.01,{ }^{* *} P<0.001$, and ${ }^{* * * *} P<0.0001$ after 2-tailed Student's $t$ test. All data are presented as mean \pm SEM.

action under high-fat conditions (Figure 6B and Supplemental Figure 7I). Male and female mice fed an HFD or SRHFD exhibited similar plasma insulin levels in the fed state (Figure 6C and Supplemental Figure 7J). To gain more insights into the insulin-sensitizing effect of selenite supplementation, we determined organ-specific insulin sensitivity in these mice after in vivo vena cava insulin infusion. Insulin injection increased $\mathrm{AKT}_{\text {Ser473 }}$ phosphorylation by $62 \%$ in skeletal muscles only in SRHFD-fed male mice compared with HFD control, while AKT activation was unaltered in liver, brown adipose tissue, subcutaneous WAT, and hypothalamus between tested groups (Supplemental Figure 8, A-J). Strikingly, insulin stimulation caused a 2.7-fold increase in $\mathrm{AKT}_{\text {Ser473 }}$ phosphorylation in gWAT of females fed an SRHFD compared with HFD control, which was to a lesser extent also present in males (Figure 6D and Supplemental Figure 8I). In line with our in vitro data, mice fed an SRHFD exhibited increased IR protein expression in gWAT, which was also present in brown adipose tissue and skeletal muscle (Figure 6D and Supplemental Figure 8, B and F). Further, we differentiated isolated stroma vascular fractions (SVFs) of gWAT from either HFD- or SRHFD-fed mice. Here, we confirmed an upregulation of Ir, Gpx3, Glut4, and $A p 2$ with a concomitant increase in Oil Red O incorporation in SRHFD-derived SVFs (Figure 6, E and F). Interestingly, differentiated SVF cells from HFD-fed mice showed a marked resistance to insulin-induced increase in ${ }^{14} \mathrm{C}-\mathrm{DOG}$ uptake, which was alleviated in SVFs from SRHFD-fed mice (Figure 6G). 
A

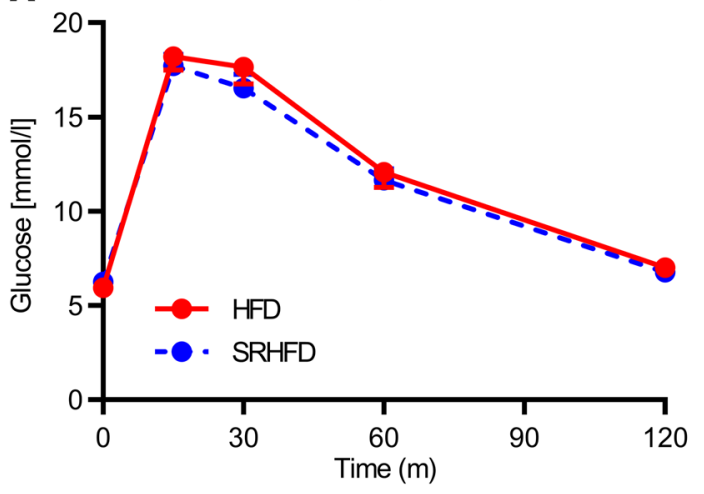

B

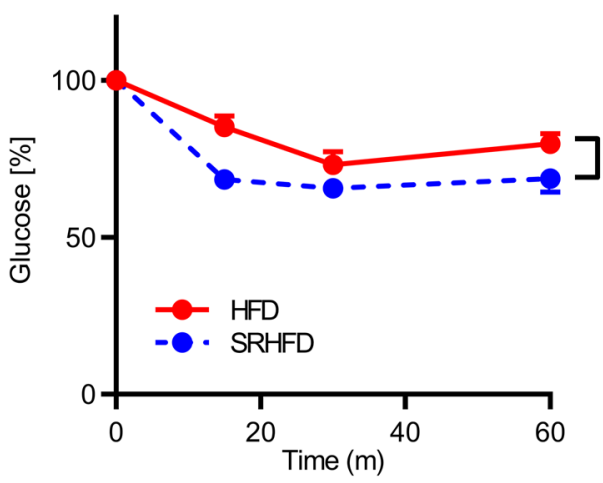

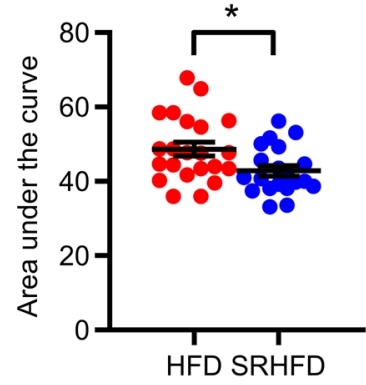

C

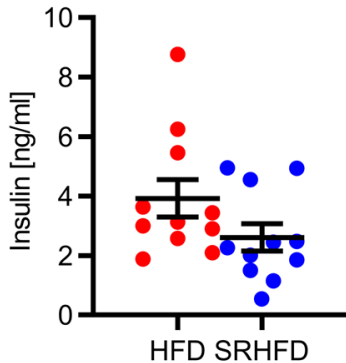

D

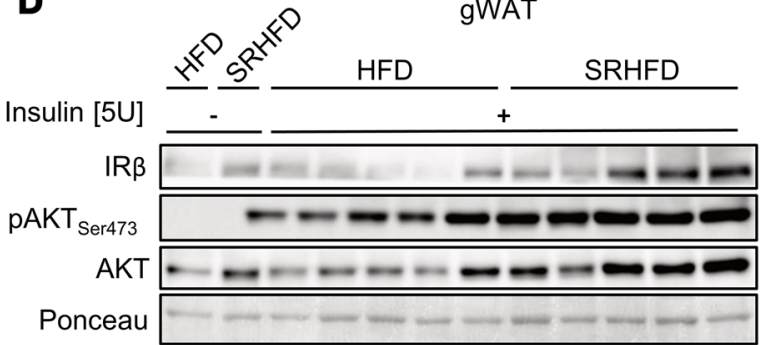

$\mathbf{F}$
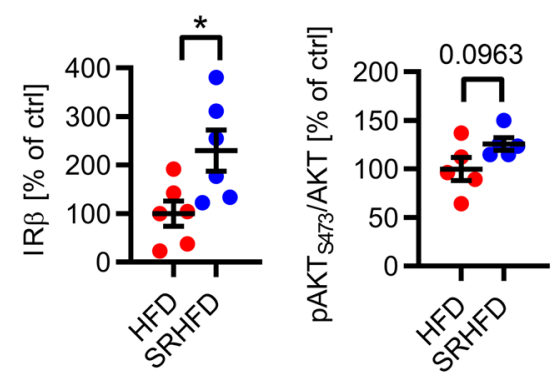

E

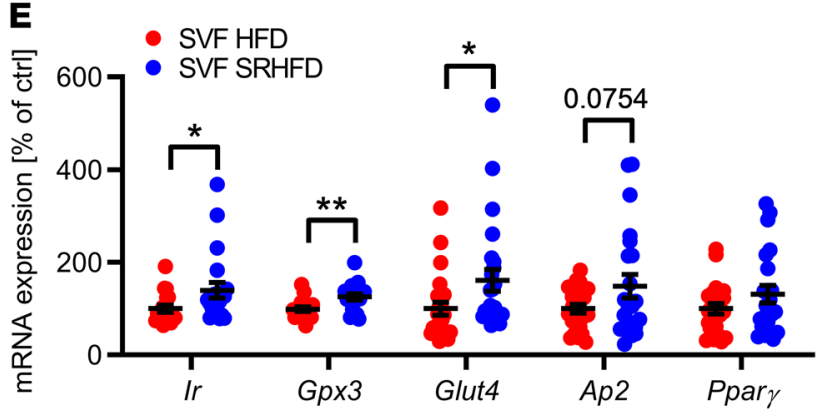

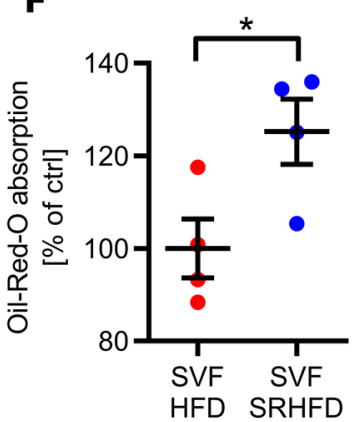

G

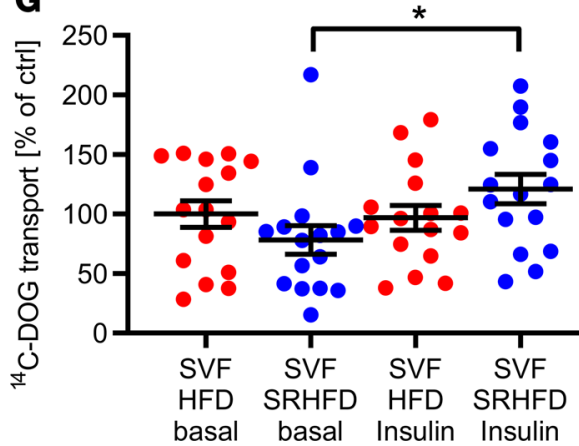

Figure 6. Selenite supplementation in diet-induced obesity attenuates insulin resistance. (A) Blood glucose levels during an oGTT of mice fed either HFD or SRHFD ( $n=12)$. (B) (Left) Blood glucose levels and (right) calculated area under the curve during an intraperitoneal ITT of mice fed either HFD or SRHFD ( $n=21$ ). (C) Plasma insulin levels in mice fed either HFD or SRHFD $(n=12)$. (D) Phosphorylation levels, protein expression, and densitometric analysis of members of the insulin signaling pathway in epigonadal WAT after bolus injection of $5 \mathrm{U}$ insulin into the vena cava. (E) Expression profile of adipocyte markers and Gpx3 of differentiated SVF-derived adipocytes from HFD- or SRHFD-fed mice $(n=16)$. (F) Oil Red 0 quantification of differentiated SVF-derived adipocytes from HFD- or SRHFD-fed mice $(n=4)$. (C) ${ }^{14} C$-DOC uptake in differentiated SVF-derived adipocytes from HFD- or SRHFD-fed mice $(n=16)$. ${ }^{*} P<0.05$, and ${ }^{* *} P<0.01$ after 2 -tailed Student's $t$ test. ${ }^{*} P<0.05$ after 2 -way ANOVA. All data are presented as mean \pm SEM.

Our data showed that HIRO causes alterations in selenoprotein mRNA expression with reduced $I r$ expression in gWAT. To identify potential selenoproteins that may regulate IR expression in selenite-treated animals, we analyzed the expression pattern of selenoproteins in gWAT of these animals. This analysis showed that there was no common up- or downregulation of the entire selenotranscriptome in mice fed either HFD or SRHFD (Figure 7A). Yet, when we compared data from Figure 1, G-I, and Figure 7A, it became apparent that specifically Gpx3, Sephs2, and Txnrd3, which were downregulated in HIRO mouse models, were upregulated in selenite-treated HFD-fed animals. In addition, mice with HIRO exhibited a lower Gpx3/Ir ratio in gWAT compared with control animals (Figure 7B). Conversely, Gpx3, Sephs2, and Txnrd3 positively correlated with $\operatorname{Ir}$ expression in murine gWAT $\left(R^{2}=0.1470, P<0.05\right.$ for $\operatorname{Ir} / G p x 3 ; R^{2}=$ 0.4577, $P<0.0001$ for $I r /$ Sephs $2 ; R^{2}=0.3614, P<0.0001$ for $I r / T x n r d 3$ ) (Figure 7, C-E). Next, we characterized this correlation in subcutaneous adipose tissue of normal-weight $(\mathrm{BMI}<25)$ and overweight/obese $(\mathrm{BMI}>25)$ patients. This analysis revealed a confirmatory lower GPX3/IR ratio and positive correlation 
of $G P X 3$ with $I R\left(R^{2}=0.2117, P<0.0001\right)$ in normal-weight compared with overweight/obese patients (Figure 7, B and F), which was not evident for SEPHS2 and TXNRD3 (correlation data not shown, ratios in Supplemental Figure 9, A-D). Furthermore, GPX3 expression in scWAT exhibited a slight but significant negative correlation with the Homeostatic Model Assessment of Insulin Resistance (HOMA-IR), indicating that GPX3 expression associates with increased insulin sensitivity (Figure 7G). This effect was also present in visceral WAT of the same patients (Supplemental Figure 9, E and F). Interestingly, we identified 2 single nucleotide polymorphisms (SNPs) in the human GPX3 gene in the database of the Meta-Analyses of Glucose and Insulin-related traits Consortium (MAGIC) (33), which were negatively associated with the insulin sensitivity index (ISI), further supporting a functional link of GPx3 and insulin action in humans (Table 1).

To functionally link these findings, we reduced GPx3 levels in 3T3-L1 preadipocytes using an siRNA knockdown approach. Strikingly, the downregulation of GPx3 caused a 30\% reduction in Ir expression. Concomitantly, decreased IR protein expression after GPx3 knockdown decreased insulin sensitivity as evidenced by reduced $\mathrm{AKT}_{\text {Ser473 }}$ phosphorylation, suggesting that selenite-induced GPx3 expression is necessary to increase IR expression and improve adipocyte function (Figure 7, H-J).

Overall, we show that HIRO is necessary for combined IR mRNA and protein downregulation in gWAT and causes reduced expression of selenoproteins GPx3, Sephs2, and Txnrd3 in gWAT. This phenotype was reversed by selenite supplementation during obesity development and counteracted insulin resistance with enhanced IR expression. We identified selenite-induced GPx3 as a novel regulator of IR expression in adipose tissue and unraveled a potentially novel interaction of selenotranscriptome homeostasis and insulin signaling in mice and humans.

\section{Discussion}

Obesity is linked to elevated oxidative stress levels and insulin resistance in adipose tissue. Though the reduction of IR expression is a common feature in diabetes, the characterization of low-grade inflammation and its negative effects on insulin signaling by posttranslational modification of insulin signaling molecules has been a major focus of research in the last decades (34). Both phenomena - stress-induced insulin resistance in adipose tissue and reduction of IR - cause metabolic disorders, highlighting the negative impact of loss of insulin signaling in adipose tissue on metabolism $(2,10)$.

Supplementation of selenium was considered to improve insulin action because it can reduce excessive oxidative stress via increased expression of selenoproteins. In addition, selenate specifically can act as an insulin mimetic $(20,35,36)$. Yet, data about insulin-sensitizing effects of selenium are controversial in regard to its potential benefits on insulin resistance in humans and animals $(37,38)$. This might be due to different amounts of supplemented selenium in combination with different baseline levels of plasma selenium in tested subjects in these trials $(39,40)$. We confirm that nanomolar concentrations of selenite supplementation increase selenoprotein expression without alterations in cellular homeostasis of 3T3-L1 preadipocytes (Supplemental Figure 2). There is only a narrow window for proper selenium and accompanying selenoprotein levels to exert beneficial health effects, and exceeding or falling below these levels might cause insulin resistance $(35,41,42)$. In line, greatly diminishing redox signaling by using antioxidants or increasing antioxidant enzymes can also be detrimental for insulin sensitivity (43). Additionally, because deficiency of the selenium transport protein SelenoP improves responsiveness to exercise and exercise-induced metabolism (44), massively elevated SelenoP levels might be detrimental for metabolism.

The fact that selenoprotein transcripts in gonadal adipose tissue were dysregulated in $\mathrm{db} / \mathrm{db}$, HFD-fed $\mathrm{C} 57 \mathrm{BL} / 6 \mathrm{~N}$, and OVX mouse models reveals that this dysregulation is independent of nutritional selenium uptake because these animals were fed the same diet (conventional NCD for $\mathrm{db} /+$ and $\mathrm{db} / \mathrm{db}$ mice, conventional $60 \% \mathrm{HFD}$ for male C57BL/6N and female sham-operated control and OVX C57BL/6N mice; see also Methods). Thus, regulation of GPx3, Sephs2, or Txnrd3 transcription in insulin-resistant states is not exclusively regulated by dietary selenium intake. It has been shown that inflammatory cytokines reduce the expression of Gpx3 in adipocytes (22). In line, we show that SRHFD reduces inflammation in gWAT, which coincides with elevated Gpx3 levels (Figure 5G). Because the expression of inflammatory markers in gWAT of HIRO mice was not uniformly affected, increased inflammation cannot exclusively explain the reduced levels of GPx3 in these mice (Supplemental Figure 10, A-D).

In this study, we identified an association of selenoprotein mRNA expression and insulin sensitivity. We show that HIRO is necessary to reduce IR expression on mRNA and protein levels in gWAT. This is linked to altered selenoprotein transcript patterns, with a common reduction of Gpx3, Sephs2, and Txnrd3 
A

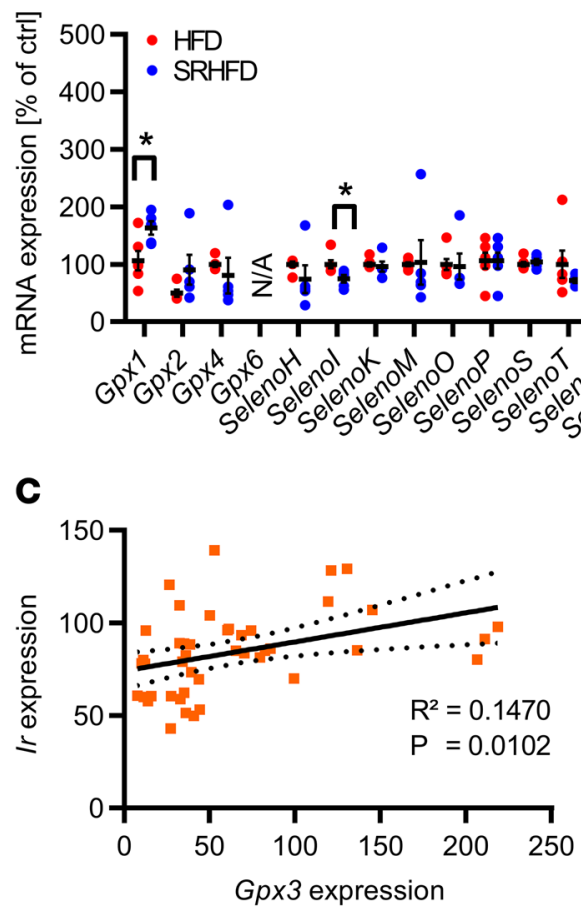

$\mathbf{F}$

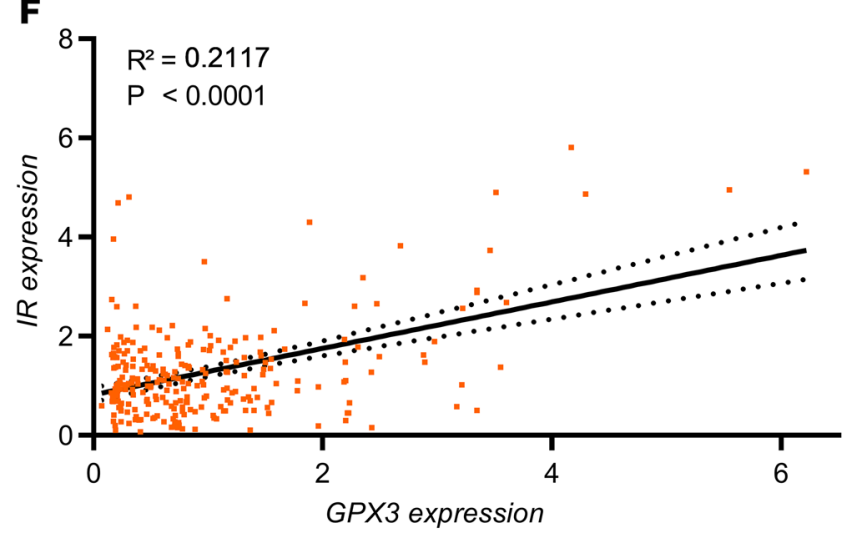

H

- NT

- Gpx3 KD

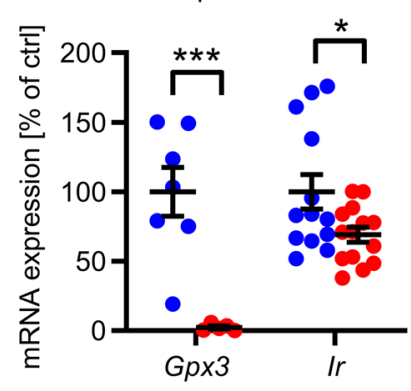

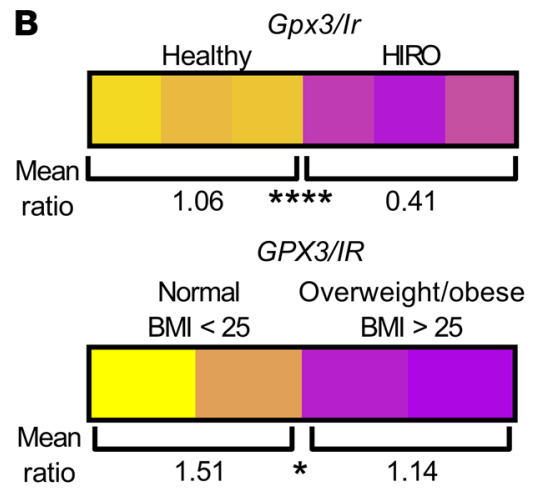

E

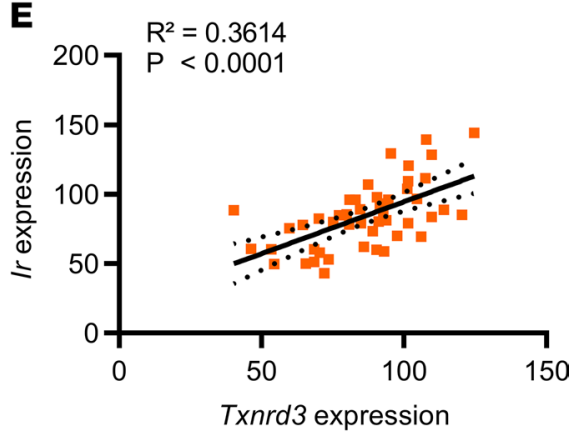

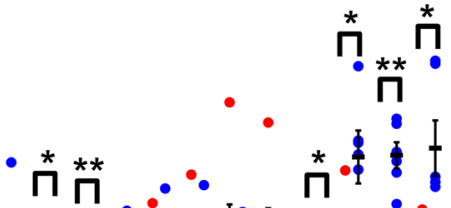

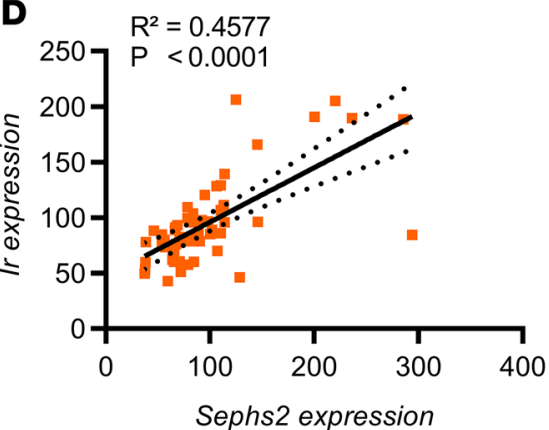

G

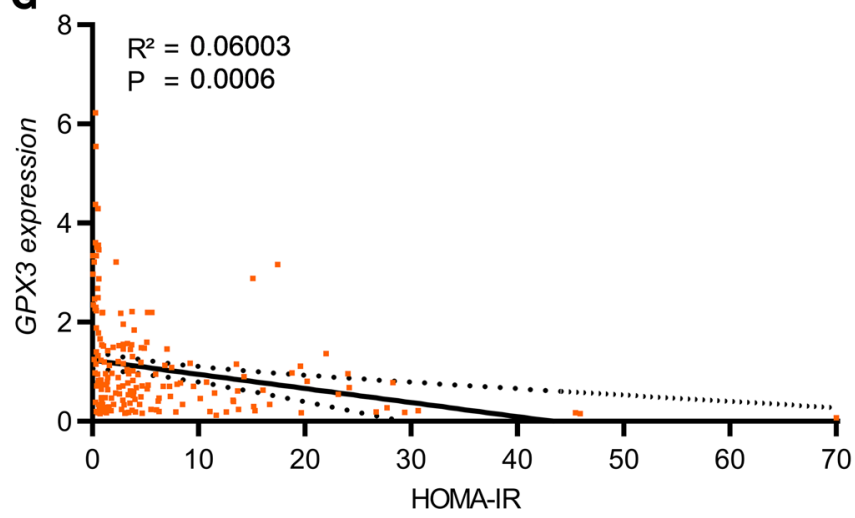

\section{I}

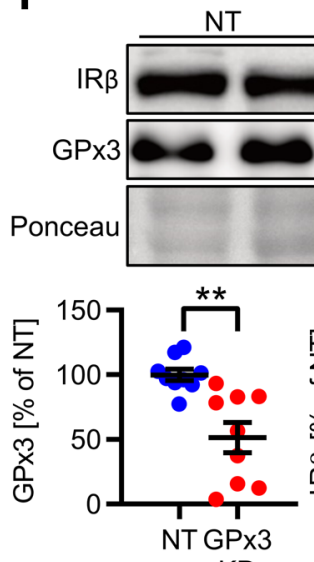

$\mathrm{KD}$

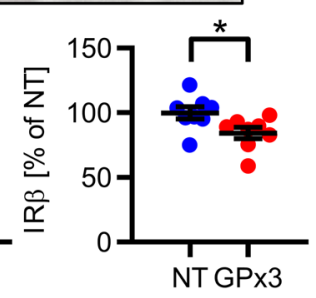

$\mathrm{KD}$
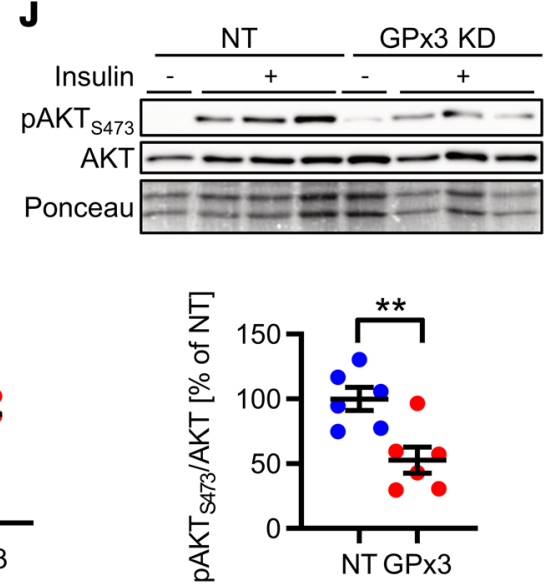

KD 
Figure 7. Gpx3 dysregulation causes insulin receptor downregulation and insulin resistance. (A) mRNA expression of the epigonadal selenoprotein transcriptome of mice after 12 weeks of either HFD or SRHFD $(n=12)$. (B) Mean Gpx3/Ir ratios in gWAT of (upper) healthy and HIRO mice ( $n=12)$ and WAT of (lower) normal-weight (BMI $<25, n=28$ ) and overweight/obese (BMI $>25, n=274$ ) human patients. (C) Correlation between Ir and Gpx3 expression in gWAT of healthy and HIRO mice $(n=6-12)$. (D and E) Correlation between Sephs2 (D) or Txnrd3 (E) with Ir expression in gWAT of healthy and HIRO mice $(n=6-12)$. (F) Correlation between IR and GPX3 in human subcutaneous WAT (scWAT) ( $n=302)$. (G) Correlation between HOMA-IR and GPX3 expression in human scWAT $(n=215)$. (H) Expression of Gpx3 and Ir after siRNA-mediated knockdown of GPX3 (NT, nontarget) in 3T3-L1 preadipocytes after selenite treatment. $n=7(G p \times 3) / 12$ (Ir). (I) Western blot of 3T3-L1 preadipocytes after siRNA mediated knockdown of GPx3 (densitometry from biological replicate experiments, $n=3$ ). (J) Western blot of insulin-stimulated 3T3-L1 preadipocytes after siRNA-mediated knockdown of GPx3 (densitometry from biological replicate experiments, $n=3$ ). Correlation calculated using Pearson's correlation coefficients; linear regression analysis was used for best-fit curve showing $95 \% \mathrm{Cl}$ in dashed lines. ${ }^{*} P<0.05,{ }^{* *} P<0.01,{ }^{* *} P<0.001$, and ${ }^{* * *} P<0.0001$ after 2 -tailed Student's $t$ test. All data are presented as mean \pm SEM.

in obese, insulin-resistant mice, with a similar GPX3 regulation in humans. In addition, we used publicly available data from the MAGIC to identify a negative association of 2 SNPs in the human GPX3 gene with the ISI. Because these SNPs were frequently present in the human population ( $41 \%$ and $45 \%$, respectively, Table 1), further research is needed to investigate the effect of these SNPs on GPx3 activity and its influence on insulin sensitivity. Moreover, 3 SNPs for GPX3 associated with the metabolic syndrome were identified in a Mexican cohort (45). These were, however, not associated with insulin sensitivity within the MAGIC analysis data set and additionally seem to be located 150-500 kb upstream of the GPX3 gene, rather than within its coding region. It needs to be stated that because of vastly different selenium supplies between different regions of the world, the association between selenium status and insulin sensitivity could be markedly different as well. Unfortunately, data about the selenium status of the patients we used for our analysis were unavailable. Still, the presented data help connect, and mechanistically link, reports about GPx3 and obesity. It has been shown that GPx3 is (a) within an adipokine cluster related to insulin sensitivity/hyperglycemia and lipid metabolism in humans (23), (b) decreased in obese patients (27), and (3) important for adipocyte function (22). Since it is known that GPx3 is a selenoprotein, this suggests that careful selenite supplementation might be beneficial for adipocyte metabolism. We show that selenite stimulation increases Gpx3 mRNA and protein levels in a dose-dependent manner and enhances $\operatorname{Ir}$ gene and protein expression in different adipocyte cell culture models (Figure 2, A-H; and Figure 6, E-G). Ir expression increases at earliest after 2 days of selenite treatment, indicating that an alteration of the selenoproteome is necessary. Interestingly, this regulation is mediated via the transcription factor Sp1, a known transcription factor controlling the expression of IR (30), while selenite-induced adipocyte differentiation stayed unaffected (Figure 2, I and J). We further show that GPx3 is important for proper selenite-induced IR signaling because GPx3 knockdown resulted in a significant reduction of IR levels and concomitantly reduced insulin action (Figure 7, H-J). This provides a putative explanation why increased GPx3 expression in $\mathrm{db} / \mathrm{db}$ mice improves metabolism (22). Because Sp1 also controls Gpx3 transcription (46), Sp1 may act via inducing GPx3 to increase IR expression above basal levels. This induction increases insulin sensitivity and adipocyte function, a phenotype also present in vivo, contributing to selenite-induced protection against diet-induced insulin resistance (see graphical abstract). Of note, although Ir mRNA levels were unaltered in our investigated ob/ob cohort compared with control, IR as well as GPx3 protein levels were reduced (Supplemental Figure 1E). These data show that decreased GPx3 protein levels in ob/ob mice cannot explain reduced IR protein levels in gonadal adipose tissue and suggest other complementary mechanisms of IR protein regulation.

Selenite-induced expression of Gpx3 cannot solely explain the sex-specific effect on adipose tissue mass in mice fed an SRHFD because GPx3-KO mice exhibit unaltered adipose tissue mass independent of sex (47). Reasons for this sex difference are still unknown but could be explained by the complex interaction of selenium status, distribution, inflammation, and sex hormones $(48,49)$. Indeed, gWAT inflammation was markedly reduced in males fed an SRHFD, but only minor changes were observed in SRHFD-fed females compared with control (Figure 5G and Supplemental Figure 7G). The effect of Sephs2 on obesity development is so far unknown but might contribute to this phenotype because Sephs 2 resides in a genetic locus that negatively correlates with body fat percentage (50). It remains unclear why selenite treatment reduces Sephs2 expression while inducing Ir expression in 3T3-L1 preadipocytes because feeding mice an SRHFD caused an increase in Sephs2 expression (Figure 7A), and knockdown of it reduced Ir expression (data not shown). This complex interaction clearly needs further investigation. The fact that 96 hours' selenite treatment reduced Sephs 2 mRNA expression in 3T3-L1 cells might be a secondary effect and related to decreased Pgcla levels (Figure 3F) because other selenoproteins have been shown to be induced by PGC1- $\alpha$ (51). 
Table 1. GPX3 SNPs negatively associated with insulin sensitivity

\begin{tabular}{|c|c|c|c|c|c|c|}
\hline SNP & Effect allele & Other allele & Effect & SEM & $P$ value & MAF \\
\hline rs8177435 & $\mathrm{T}$ & G & $-2.3 e-02$ & $1.1 e-02$ & 0.04195 & $T=0.4107 / 2057$ \\
\hline rs8177431 & $A$ & $G$ & $-2.3 e-02$ & $1.1 e-02$ & 0.04218 & $A=0.4453 / 2230$ \\
\hline
\end{tabular}

Identities, effects, and minor allele frequencies (MAFs, 1000 Genomes Project) of GPX3 SNPs associated with a reduced ISI.

Interestingly, mice fed an SRHFD exhibited adipocyte hypertrophy compared with the control group, and gWAT exhibited decreased inflammation and enhanced insulin action. Thus, selenite-induced adipocyte hypertrophy is different from classical unhealthy adipocyte expansion and implies that adipocyte hypertrophy does not have to be detrimental for adipose tissue expansion and insulin sensitivity (52). Our data contrast a recent study showing that comparable selenite stimulation did not exert similar beneficial effects on adipocyte differentiation in vitro (21). One possible contributor could be the inherent selenium content of the respective fetal bovine serum (FBS) used in the cell culture medium because selenium content can differ substantially depending on the origin of the FBS, therefore leading to different baseline selenium statuses than we used in our experiments.

The selenite-induced regulation of Ir expression was specific for gWAT. Although Ir expression was also increased in skeletal muscle and brown adipose tissue, treating myoblasts or brown preadipocytes with selenite did not alter Ir expression (Supplemental Figure 11, A and B). Our results suggest that the observed elevated IR levels in these tissues are secondary to selenite supplementation and might be a consequence of overall improved insulin sensitivity. The question remains why selenite treatment increased IR expression only in gWAT.

The identification of GPx3 as mediator of IR expression is of importance because dietary selenium supplementation revealed controversial results with regard to beneficial effects on metabolism (53). Thus, identifying novel compounds that induce GPx3 in diabetic patients with normal selenium status might help improve metabolism and counteract adipocyte dysfunction. This approach might help replace selenite supplementation as a means to induce GPx3, avoiding the difficulty of a fine-tuned safe selenium supplementation. Of note, it has been already shown that insulin sensitizers thiazolidinediones increase Gpx3 expression in skeletal muscle (54).

Overall our data reveal a potentially novel function of the selenotranscriptome in controlling IR expression via GPx3 and enhancing gonadal adipose tissue insulin sensitivity to improve metabolism and counteract diet-induced insulin resistance.

\section{Methods}

Animal studies. C57BL/6N mice were bred in-house and group housed in a temperature-controlled room $\left(22 \pm 1^{\circ} \mathrm{C}\right.$ ) on a 12-hour light/12-hour dark cycle with free access to food and water. The experimental diets for selenium supplementation were custom made, contained $60 \%$ of calories from fat, and differed in selenium content (HFD: $230 \mathrm{ng} / \mathrm{g}$ Se and SRHFD: $676 \mathrm{ng} / \mathrm{g}$, Supplemental Table 1) (ssniff Spezialdiäten $\mathrm{GmbH}$, Germany). Animals were kept on diets for 12 weeks, starting at 4 weeks of age. ITT was performed with an intraperitoneal injection of insulin ( $0.75 \mathrm{U} / \mathrm{kg}$ body weight). For the glucose tolerance test, animals were starved for 16 hours, and 2 g glucose $/ \mathrm{kg}$ body weight was applied orally. Energy expenditure and respiratory quotient were measured via indirect calorimetry using the PhenoMaster (TSE Systems GmbH, Germany). Body composition was determined via NMR spectroscopy using the EchoMRI (EchoMRI LLC, USA). To assess local insulin sensitivity, mice were kept on either diet for 12 weeks, starved for 16 hours, and anesthetized. Then, $5 \mathrm{U}$ of insulin was injected directly into the vena cava. Organs were harvested after the time points in parentheses: Liver ( 2 minutes), quadriceps ( 5 minutes), gonadal and subcutaneous WAT (5 minutes), and brain (10 minutes). For diet-induced obesity and long-term insulin resistance, C57BL/6N mice were fed either NCD (300 ng/g selenium, ssniff Spezialdiäten GmbH, Germany) or a conventional 60\% HFD (300 ng/g selenium, ssniff Spezialdiäten GmbH, Germany) for 3 days or 12 weeks, starting at 4 weeks of age. Ovariectomies (OVX) or sham surgeries were performed at 4 weeks of age under deep anesthesia. Through dorsal incisions the ovaries were accessed and cauterized. After a 1-week regeneration period, sham or OVX mice were fed a 60\% HFD for 14 weeks. Male ob/+, ob/ob (B6.Cg-Lep $\left.{ }^{\mathrm{ob}} / \mathrm{J}\right)$, 
$\mathrm{db} /+$, and $\mathrm{db} / \mathrm{db}\left(\right.$ BKS.Cg-Dock $7^{\mathrm{m}}+/+\mathrm{Lepr}^{\mathrm{db}} / \mathrm{J}$ ) mice were purchased at 10 weeks of age (Charles River Laboratories Inc., USA), kept on NCD (see above) for 2 weeks, and euthanized at 12 weeks of age.

Isolation of stroma vascular fraction. Gonadal adipose tissue of C57BL/6N mice fed HFD or SRHFD for 12 weeks was collected, digested with Collagenase II, and filtered through a $50-\mu \mathrm{m}$ mesh, and the resulting SVF cells were plated at a density of $2.5 \times 10^{4}$ cells $/ \mathrm{cm}^{2}$. Differentiation was induced as described below.

Cell culture. White 3T3-L1 preadipocyte cells were cultured in low-selenium culture medium (condition C), Gibco DMEM GlutaMAX (Thermo Fisher Scientific Inc., USA), supplemented with 10\% FBS (containing a final concentration of $9.7 \mathrm{nM}$ selenium in the medium), 1\% penicillin/streptomycin (Thermo Fisher Scientific Inc., USA), and 1\% pyruvate (Thermo Fisher Scientific Inc., USA) at $37^{\circ} \mathrm{C}$ and $5 \%$ $\mathrm{CO}_{2}$. For selenium-treated cells we used the same medium supplemented with $200 \mathrm{nM}$ sodium selenite (condition Se) 3T3-L1 differentiation, in the absence (C) or presence of $200 \mathrm{nM}$ sodium selenite (Se), and Oil Red O staining was performed as previously described (55). For acute insulin stimulation cells were serum starved for 3 hours and stimulated with $100 \mathrm{nM}$ insulin for 5 minutes. Glucose uptake was measured using ${ }^{14} \mathrm{C}$-DOG. In brief, cells were serum starved for 6 hours and incubated for 20 minutes with either $250 \mathrm{nM}$ insulin or $10 \mu \mathrm{M}$ Cytochalasin B (see Supplemental Table 6 for the manufacturer of this and other compounds.) before addition of $2 \mathrm{mM}{ }^{14} \mathrm{C}$-DOG for 10 minutes. Cells were then lysed and incubated with Ultima Gold scintillation liquid (PerkinElmer Inc., USA). Samples were measured with the Beckman Coulter LS6500 Liquid Scintillation Counter. Knockdown experiments with smartPOOL siRNA (Gpx3: M-042578-01, Sephs2: M-050751-01, nontarget: D-001810-01-05, Horizon Discovery Ltd., USA) were performed according to the manufacturer's guidelines.

Analytical procedures. Glucose was measured with a Glucometer - Contour XT (Bayer AG, Germany). Insulin was measured with the ELISA Kit from ALPCO (ALPCO BioCat GmbH, Switzerland). Leptin was measured using an ELISA from R\&D Systems (Bio-Techne $\mathrm{GmbH}$, Germany). All ELISAs were performed according to manufacturers' recommendations. Triacylglycerols were measured with Triglyceride Reagent from HORIBA ABX SAS (Japan), and NEFAs were measured with NEFA-HR Assay according to the manufacturer's instructions (FUJIFILM Wako Chemicals Europe GmbH, Germany). For assessment of selenite-induced effects on cell membrane integrity, 3T3-L1 preadipocytes were cultured as described above. Cells were incubated with PBS or sodium selenite in PBS at $200 \mathrm{nM}, 500 \mathrm{nM}, 1000 \mathrm{nM}, 5000 \mathrm{nM}$, or 10,000 nM for 96 hours. Thereafter, supernatants were isolated and stored at $-20^{\circ} \mathrm{C}$ until further analyses. LDH activity as a marker for necrosis was measured by a UV test (Roche Diagnostics $\mathrm{GmbH}$ ). Lactate was determined by an enzymatic color test (Roche Diagnostics $\mathrm{GmbH}$ ).

GPx activity assay. Mouse tissue or cell pellets were homogenized, lysed by sonication, and centrifuged. GPx activity was measured using a glutathione reductase coupled test with $\mathrm{H}_{2} \mathrm{O}_{2}$ as substrate and normalized to protein content (56).

Immunohistochemistry. Fresh tissue samples were submerged in 4\% paraformaldehyde for 24 hours at $4^{\circ} \mathrm{C}$, washed in PBS, and embedded in paraffin. Slices were fixed on glass slides and stained with hematoxylin and eosin. Calculations of adipocyte size were performed with ImageJ Fiji (NIH, USA) (57).

$R N A / D N A$ isolation. Total RNA from tissue was extracted using the RNeasy Mini Kit (QIAGEN $\mathrm{GmbH}$, Germany) or ReliaPrep RNA Tissue Miniprep System (Promega Corporation, USA) including DNase I treatment following the manufacturer's manual. Total RNA from cells was isolated using phenol-chloroform extraction. DNA from cells was extracted using the Invisorb Spin Tissue Mini Kit (Invitek Molecular $\mathrm{GmbH}$, Germany) following the manufacturer's manual.

Analysis of gene expression by quantitative real-time PCR. RNA from tissue or cells was reverse-transcribed using oligo(dT)15 primers (Promega Corporation, USA), random primers (Promega Corporation), Thermo Fisher Scientific dNTP-Set, and M-MLV reverse transcriptase (Promega Corporation). Real-time quantitative PCR was performed with $10 \mathrm{ng}$ cDNA (for Gpx3 detection in 3T3-L1 cells specifically, $20 \mathrm{ng}$ had to be used) using GoTaq qPCR Master Mix (Promega Corporation) and gene-specific primers (Supplemental Table 3). Fluorescence was analyzed in the ViiA-7-Real-Time-PCR System (Applied Biosystems, Thermo Fisher Scientific Inc., USA). Gene expression was calculated applying $\Delta \Delta C T$ method using TATA binding protein as reference gene. For mitochondrial DNA content choline dehydrogenase 1 was used as reference gene. The specificity of primers was confirmed by melting curve analysis.

Western blot analysis. Protein samples were separated via SDS-PAGE and transferred to Amersham Hybond PVDF membranes (VWR International, LLC, USA). Membranes were blocked in StartingBlock T20 Blocking Buffer (Thermo Fisher Scientific Inc., USA), probed with primary antibody (Supplemental Table 4), 
and then incubated with peroxidase-conjugated secondary antibodies (Supplemental Table 5). Bands were detected via chemiluminescence (WesternBright ECL Biozym Scientific, Germany) using the ChemiDoc Touch Imaging System (Bio-Rad Laboratories, Inc, USA). Ponceau staining was used as loading control. Band intensities were quantified via densitometric analysis using ImageJ Fiji.

Protein carbonylation. Carbonylated proteins were detected as previously described (58). Briefly, membranes were incubated for derivatization with $20 \mathrm{mM}$ 2,4-dinitrophenylhydrazine in $2 \mathrm{M} \mathrm{HCl}$ and afterward probed with anti-DNP primary antibody (Merck KGaA, Germany).

Seahorse assay. Mitochondrial respiration was monitored using Seahorse XF Mito Stress Test Kit and Seahorse XF96 Extracellular Flux Analyzer (Agilent Technologies, Inc., USA), measuring oxygen consumption rate (OCR) of adherent cells to monitor mitochondrial function. Final compound concentrations were $2 \mu \mathrm{M}$ oligomycin, $1 \mu \mathrm{M}$ FCCP, and $1 \mu \mathrm{M} / 2 \mu \mathrm{M}$ rotenone/antimycin A. For the permeabilization assay cells were pretreated with $50 \mu \mathrm{g} / \mathrm{mL}$ saponin. The assay was otherwise performed according to manufacturer's instructions. OCR values were normalized to total protein content.

Inductively coupled plasma-mass spectrometry (ICP-MS). Quantification of total selenium in cells, adipose tissue, feed, and brain samples was performed as follows. Cells were incubated with the respective medium for 24 to 96 hours. Brain and adipose tissue samples of mice, fed either HFD or SRHFD for 12 weeks, were homogenized using mortar and pestle in liquid nitrogen. Afterward, cell pellets or brain homogenates were digested with nitric acid using a microwave digestion system (MARS 6, CEM Corporation, USA). In the case of cell pellets, samples were resuspended in $470 \mu \mathrm{L}$ deionized water and transferred to a trifluoroacetic acid (TFA) microwave vessel with an additional $455 \mu \mathrm{L}$ of deionized water. Afterward, $500 \mu \mathrm{L}$ of concentrated nitric acid (65\%, suprapure, Merck KGaA, Germany) and $75 \mu \mathrm{L}$ of $100 \mu \mathrm{g} / \mathrm{L}{ }^{77} \mathrm{Se}(3 \mu \mathrm{g} / \mathrm{L})$ as isotope dilution standard (diluted from dissolved isotopically enriched standard $\left[97.20 \pm 0.20 \%{ }^{77} \mathrm{Se} ; 0.10 \%{ }^{74} \mathrm{Se}\right.$; $\left.0.40 \pm 0.10 \%{ }^{76} \mathrm{Se} ; 2.40 \pm 0.10 \%{ }^{78} \mathrm{Se} ; 0.10 \%{ }^{80} \mathrm{Se} ; 0.10 \%{ }^{82} \mathrm{Se}\right]$ as certified by Trace Sciences International (Canada), purchased from Eurisotop SAS (Germany) were added to the same microwave vessel. For feed and brain samples, approximately $50 \mathrm{mg}$ of sample was weighed into microwave TFA vessels. Then, 900 $\mu \mathrm{L} \mathrm{HNO}_{3}(65 \%), 250 \mu \mathrm{L} \mathrm{H}_{2} \mathrm{O}_{2}\left(30 \%\right.$ Merck KGaA, Germany), and $30 \mu \mathrm{L}$ of ${ }^{77} \mathrm{Se}(1000 \mu \mathrm{g} / \mathrm{L})$ as well as $820 \mu \mathrm{L}$ of deionized water were added. For all sample types, digestion was carried out by heating to $200^{\circ} \mathrm{C}$ within 15 minutes in closed microwave vessels. This temperature was maintained for another 20 minutes. After cooling the temperature, $1 \mathrm{~mL}$ of deionized water was added to the cell pellet digests, and this solution was transferred to $15-\mathrm{mL}$ plastic tubes for measurement via ICP-MS. The same protocol was applied to $12.8 \mathrm{mg}$ of a certified reference material (BCR-274, Single Cell Protein CRM JRC with $1.03 \mu \mathrm{g} / \mathrm{g}$ Se) for validation (recovery: 104.9\%). For brain sample digests, $2 \mathrm{~mL}$ of deionized water was added, and the solution was transferred to $15-\mathrm{mL}$ plastic vessels. The solution was then further diluted 1:4 with deionized water before ICP-MSMS measurement for brain samples. In the case of feed samples, the solution was further diluted 1:1 using a solution containing the same concentration of nitric acid and internal standards as in the digest solutions. For brain and feed samples, approximately $50 \mathrm{mg}$ of certified reference materials ERM-BB 422 — fish muscle (c[Se]: $1.33 \mathrm{mg} / \mathrm{kg}$ ) — and ERM-BB 186 — pig kidney (c[Se]: $10.3 \mathrm{mg} / \mathrm{kg})$ were subjected to the same protocol for validation (recoveries: $109.4 \%$ and $103.9 \%$, respectively).

Afterward, the selenium quantification was carried out using ICP-MSMS (Agilent 8800, Agilent Technologies, Germany). Selenium was measured in oxygen mass-shift mode on isotopes $77 \rightarrow 93$ and $80 \rightarrow 96$ with an integration time of 0.5 seconds and 3 replicates per isotope. The signal ratio of these isotopes was then used to calculate selenium concentrations via isotope dilution analysis. Instrument parameters were as follows: plasma Rf power: $1550 \mathrm{~W}$, plasma gas flow: $15.0 \mathrm{~L} / \mathrm{min}$, nebulizer: MicroMist at $1.20 \mathrm{~L} / \mathrm{min} \mathrm{Ar}$, spray chamber: Scott Type at $2^{\circ} \mathrm{C}$, Cones: $\mathrm{Ni}$, and cell gas: $0.6 \mathrm{~mL} / \mathrm{min} \mathrm{O}_{2}$. The instrument was tuned on a daily basis for maximum sensitivity on ${ }^{80 \rightarrow 96} \mathrm{Se}$ and a doubly charged ratio less than $2 \%\left({ }^{140} \mathrm{Ce}^{2+} /{ }^{140} \mathrm{Ce}^{+}\right)$as well as a background of less than 0.1 counts per second.

Total reflection $x$-ray fluorescence. Liver tissue of mice fed either HFD or SRHFD for 12 weeks was digested under acidic conditions using a Speedwave Two microwave (Berghof, Germany). Yttrium was used as a digestion control ( $1 \mathrm{mg} / \mathrm{L}$, Merck KGaA, Germany). Serum samples were analyzed without digestion. Afterward, a gallium standard $(0.5 \mathrm{mg} / \mathrm{L}$, Thermo Fisher Scientific, Inc., USA) was added to the sample, and $10 \mu \mathrm{L}$ of the samples was placed on siliconized quartz glass sample carriers and dried overnight. To analyze total Se content, bench-top total reflection x-ray fluorescence spectrometer (S2 Picofox, Bruker Nano GmbH, Germany) was used. Samples were measured in triplicates for 1000 seconds, and pig kidney (European Commission JRC, IRM, Belgium) was used as certified reference material for method validation. 
Human studies. In a cross-sectional study of 302 individuals (205 women, 97 men; BMI range: $16.9-$ $85.5 \mathrm{~kg} / \mathrm{m}^{2}$; age range: 16-90 years; clinical parameters in Supplemental Table 2), we investigated GPX3 and $I R$ mRNA expression in paired abdominal omental and subcutaneous WAT samples collected during elective laparoscopic abdominal surgery as described previously. Adipose tissue was immediately frozen in liquid nitrogen and stored at $-80^{\circ} \mathrm{C}$. Measurement of body composition and metabolic parameters was performed as described previously $(27,59)$.

Analysis of GPX3 and IR mRNA expression in human WAT. RNA from adipose tissue was extracted by using RNeasy Lipid Tissue Mini Kit (QIAGEN, Germany). Quantity and integrity of RNA were monitored with NanoVue Plus Spectrophotometer (GE Healthcare, Germany). One microgram total RNA from subcutaneous and visceral adipose tissue were reverse-transcribed with standard reagents (Life Technologies, Germany). cDNA was then processed for TaqMan probe-based quantitative real-time polymerase chain reaction (qPCR) using the QuantStudio 6 Flex Real-Time PCR System (Life Technologies). Expression of GPX3 and $I R$ was calculated by standard curve method and normalized to the expression of hypoxanthine guanine phosphoribosyltransferase 1 (HPRT1) as a housekeeping gene. The probes (Life Technologies) for GPX3 (Hs01078668_m1), IR (Hs00961557_m1), and HPRT1 (Hs01003267_m1) span exon-exon boundaries to improve the specificity of the qPCR. In a subset of patients, SEPHS2 and TXNRD3 mRNA expression was analyzed using Illumina human HT-12 expression chips (Illumina, Inc., USA) (normal weight $n=18$, overweight/obese $n=37$ ).

Identification of SNPS. SNPs were identified in the publicly available data from www.magicinvestigators. org, specifically the MAGIC Model 3 JMA data set from Walford et al. (33).

Data and resource availability. All data generated or analyzed during this study are included in the published article (and its online supplement). No applicable resources were generated during the current study.

Statistics. All data are presented as mean \pm SEM. Groups were compared using unpaired 2-tailed Student's $t$ test. Two-way ANOVA was performed to detect interactions between e.g., sex and treatment (diet), and Holm-Šídák post hoc analysis was performed when appropriate. Correlations in mice and humans were conducted using linear regression models. All in vitro experiments were performed in independent triplicates. Statistical analysis and generation of graphs were done using GraphPad Prism 7 (GraphPad Software Inc., USA).

Study approval. All animal procedures were conducted according to German laws for the protection of animals and in compliance with protocols approved by local government authorities (State Agency of Environment, Health and Consumer Protection, State of Brandenburg, Germany). The human study was approved by the Ethics Committee of the University of Leipzig (approval 159-12-21052012) and performed in accordance to the Declaration of Helsinki. All subjects gave written informed consent before taking part in this study.

\section{Author contributions}

$\mathrm{RH}$ designed the study, researched data, and wrote the manuscript. VS, CC, TF, MR, KR, MS, KW, SD, MS, JFK, MB, KK, TS, and APK researched data and helped design experiments. AK designed the study, supervised all work, and wrote the manuscript. AK is the guarantor of this work and, as such, had full access to all the data in the study and takes responsibility for the integrity of the data and the accuracy of the data analysis.

\section{Acknowledgments}

We thank Wenke Jonas for assistance with the indirect calorimetry and Annett Holms for technical assistance. Data on SNPs and glycemic traits have been contributed by MAGIC investigators and have been downloaded from www.magicinvestigators.org. This work was supported by the Deutsche Forschungsgemeinschaft (DFG) grant project KL 2399/4-1 (to AK) and by a grant from the German Ministry of Education and Research and the State of Brandenburg (DZD grant 82DZD00302), as well as a DFG grant CRC 1052 (project number 209933838, subproject B1 to MB).

Address correspondence to: André Kleinridders, Department of Molecular and Experimental Nutritional Medicine, University of Potsdam, Arthur-Scheunert-Allee 114-116, 14558 Nuthetal, Germany. Phone: 49.33200.885230; Email: kleinridders@uni-potsdam.de. 
1. Gregor MF, Hotamisligil GS. Inflammatory mechanisms in obesity. Annu Rev Immunol. 2011;29:415-445.

2. Wellen KE, Hotamisligil GS. Inflammation, stress, and diabetes. J Clin Invest. 2005;115(5):1111-1119.

3. Hayes GR, Lockwood DH. Role of insulin receptor phosphorylation in the insulinomimetic effects of hydrogen peroxide. Proc Natl Acad Sci U S A. 1987;84(22):8115-8119.

4. Castro JP, Grune T, Speckmann B. The two faces of reactive oxygen species (ROS) in adipocyte function and dysfunction. Biol Chem. 2016;397(8):709-724.

5. Wigand JP, Blackard WG. Downregulation of insulin receptors in obese man. Diabetes. 1979;28(4):287-291.

6. Ott R, et al. Reduced insulin receptor expression and altered DNA methylation in fat tissues and blood of women with GDM and offspring. J Clin Endocrinol Metab. 2019;104(1):137-149.

7. Freychet $\mathrm{P}$, et al. Impairment of insulin binding to the fat cell plasma membrane in the obese hyperglycemic mouse. FEBS Lett. 1972;25(2):339-342.

8. Olefsky JM. Decreased insulin binding to adipocytes and circulating monocytes from obese subjects. J Clin Invest. 1976;57(5):1165-1172.

9. Guilherme A, Virbasius JV, Puri V, Czech MP. Adipocyte dysfunctions linking obesity to insulin resistance and type 2 diabetes Nat Rev Mol Cell Biol. 2008;9(5):367-377.

10. Boucher J, et al. Differential roles of insulin and IGF-1 receptors in adipose tissue development and function. Diabetes. 2016;65(8):2201-2213.

11. Hill KE, Zhou J, McMahan WJ, Motley AK, Burk RF. Neurological dysfunction occurs in mice with targeted deletion of the selenoprotein P gene. J Nutr. 2004;134(1):157-161.

12. Ingold I, et al. Selenium utilization by GPX4 is required to prevent hydroperoxide-induced ferroptosis. Cell. 2018;172(3):409422.e21.

13. Loh K, et al. Reactive oxygen species enhance insulin sensitivity. Cell Metab. 2009;10(4):260-272.

14. Pitts MW, et al. Deletion of selenoprotein M leads to obesity without cognitive deficits. J Biol Chem. 2013;288(36):26121-26134

15. Stoffaneller R, Morse NL. A review of dietary selenium intake and selenium status in Europe and the Middle East. Nutrients. 2015;7(3):1494-1537.

16. Alasfar F, Ben-Nakhi M, Khoursheed M, Kehinde EO, Alsaleh M. Selenium is significantly depleted among morbidly obese female patients seeking bariatric surgery. Obes Surg. 2011;21(11):1710-1713.

17. Błażewicz A, et al. Serum and urinary selenium levels in obese children: a cross-sectional study. J Trace Elem Med Biol. 2015;29:116-122

18. Zeng MS, et al. A high-selenium diet induces insulin resistance in gestating rats and their offspring. Free Radic Biol Med. 2012;52(8):1335-1342.

19. Vinceti M, Filippini T, Rothman KJ. Selenium exposure and the risk of type 2 diabetes: a systematic review and meta-analysis. Eur J Epidemiol. 2018;33(9):789-810.

20. Ezaki O. The insulin-like effects of selenate in rat adipocytes. J Biol Chem. 1990;265(2):1124-1128.

21. Steinbrenner H, Micoogullari M, Hoang NA, Bergheim I, Klotz LO, Sies H. Selenium-binding protein 1 (SELENBP1) is a marker of mature adipocytes. Redox Biol. 2019;20:489-495.

22. Lee YS, et al. Dysregulation of adipose glutathione peroxidase 3 in obesity contributes to local and systemic oxidative stress Mol Endocrinol. 2008;22(9):2176-2189.

23. Flehmig G, et al. Identification of adipokine clusters related to parameters of fat mass, insulin sensitivity and inflammation. PLoS One. 2014;9(6):e99785

24. Mamula PW, et al. Regulating insulin-receptor-gene expression by differentiation and hormones. Diabetes Care. 1990;13(3):288-301.

25. Puig O, Tjian R. Transcriptional feedback control of insulin receptor by dFOXO/FOXO1. Genes Dev. 2005;19(20):2435-2446.

26. Gonzalez E, Flier E, Molle D, Accili D, McGraw TE. Hyperinsulinemia leads to uncoupled insulin regulation of the GLUT4 glucose transporter and the FoxO1 transcription factor. Proc Natl Acad Sci U S A. 2011;108(25):10162-10167.

27. Langhardt $J$, et al. Effects of weight loss on glutathione peroxidase 3 serum concentrations and adipose tissue expression in human obesity. Obes Facts. 2018;11(6):475-490.

28. Wardelmann $\mathrm{K}$, et al. Insulin action in the brain regulates mitochondrial stress responses and reduces diet-induced weight gain. Mol Metab. 2019;21:68-81.

29. Ottaviano FG, Tang SS, Handy DE, Loscalzo J. Regulation of the extracellular antioxidant selenoprotein plasma glutathione peroxidase (GPx-3) in mammalian cells. Mol Cell Biochem. 2009;327(1-2):111-126.

30. Chen H, Walker GE, Taylor SI, McKeon C. Proximal enhancer of the human insulin receptor gene binds the transcription fac tor Sp1. Diabetes. 1994;43(7):884-889.

31. Vernochet $C$, et al. Adipose tissue mitochondrial dysfunction triggers a lipodystrophic syndrome with insulin resistance, hepatosteatosis, and cardiovascular complications. FASEB J. 2014;28(10):4408-4419.

32. Greenberg AS, Obin MS. Obesity and the role of adipose tissue in inflammation and metabolism. Am J Clin Nutr. 2006;83(2):461S-465S

33. Walford GA, et al. Genome-wide association study of the modified stumvoll insulin sensitivity index identifies BCL2 and FAM19A2 as novel insulin sensitivity loci. Diabetes. 2016;65(10):3200-3211.

34. Boucher J, Kleinridders A, Kahn CR. Insulin receptor signaling in normal and insulin-resistant states. Cold Spring Harb Perspect Biol. 2014;6(1):a009191.

35. Wrobel JK, Power R, Toborek M. Biological activity of selenium: revisited. IUBMB Life. 2016;68(2):97-105

36. Stapleton SR. Selenium: an insulin-mimetic. Cell Mol Life Sci. 2000;57(13-14):1874-1879.

37. Zhou J, et al. Selenite exacerbates hepatic insulin resistance in mouse model of type 2 diabetes through oxidative stress-mediated JNK pathway. Toxicol Appl Pharmacol. 2015;289(3):409-418.

38. Steinbrenner H, Speckmann B, Pinto A, Sies H. High selenium intake and increased diabetes risk: experimental evidence for interplay between selenium and carbohydrate metabolism. J Clin Biochem Nutr. 2011;48(1):40-45.

39. Farrokhian A, et al. Selenium supplementation affects insulin resistance and serum hs-CRP in patients with type 2 diabetes and coronary heart disease. Horm Metab Res. 2016;48(4):263-268. 
40. Stranges S, et al. Effects of long-term selenium supplementation on the incidence of type 2 diabetes: a randomized trial. Ann Intern Med. 2007;147(4):217-223.

41. Steinbrenner H. Interference of selenium and selenoproteins with the insulin-regulated carbohydrate and lipid metabolism. Free Radic Biol Med. 2013;65:1538-1547.

42. Labunskyy VM, Lee BC, Handy DE, Loscalzo J, Hatfield DL, Gladyshev VN. Both maximal expression of selenoproteins and selenoprotein deficiency can promote development of type 2 diabetes-like phenotype in mice. Antioxid Redox Signal. 2011;14(12):2327-2336.

43. Tiganis T. Reactive oxygen species and insulin resistance: the good, the bad and the ugly. Trends Pharmacol Sci. 2011;32(2):82-89.

44. Misu H, et al. Deficiency of the hepatokine selenoprotein P increases responsiveness to exercise in mice through upregulation of reactive oxygen species and AMP-activated protein kinase in muscle. Nat Med. 2017;23(4):508-516.

45. Baez-Duarte BG, et al. Glutathione peroxidase 3 serum levels and GPX3 gene polymorphisms in subjects with metabolic syndrome. Arch Med Res. 2014;45(5):375-382.

46. Bierl C, Voetsch B, Jin RC, Handy DE, Loscalzo J. Determinants of human plasma glutathione peroxidase (GPx-3) expression. J Biol Chem. 2004;279(26):26839-26845.

47. Yang X, et al. Validation of candidate causal genes for obesity that affect shared metabolic pathways and networks. Nat Genet. 2009;41(4):415-423.

48. Zhou X, Smith AM, Failla ML, Hill KE, Yu Z. Estrogen status alters tissue distribution and metabolism of selenium in female rats. J Nutr Biochem. 2012;23(6):532-538.

49. Stoedter M, Renko K, Hög A, Schomburg L. Selenium controls the sex-specific immune response and selenoprotein expression during the acute-phase response in mice. Biochem J. 2010;429(1):43-51.

50. Parks BW, et al. Genetic control of obesity and gut microbiota composition in response to high-fat, high-sucrose diet in mice. Cell Metab. 2013;17(1):141-152.

51. Speckmann B, et al. Selenoprotein P expression is controlled through interaction of the coactivator PGC-1alpha with FoxO1a and hepatocyte nuclear factor 4alpha transcription factors. Hepatology. 2008;48(6):1998-2006.

52. Sun K, Kusminski CM, Scherer PE. Adipose tissue remodeling and obesity. J Clin Invest. 2011;121(6):2094-2101.

53. Rayman MP, Stranges S. Epidemiology of selenium and type 2 diabetes: can we make sense of it? Free Radic Biol Med. 2013;65:1557-1564.

54. Chung SS, et al. Glutathione peroxidase 3 mediates the antioxidant effect of peroxisome proliferator-activated receptor gamma in human skeletal muscle cells. Mol Cell Biol. 2009;29(1):20-30.

55. Ussar S, Bezy O, Blüher M, Kahn CR. Glypican-4 enhances insulin signaling via interaction with the insulin receptor and serves as a novel adipokine. Diabetes. 2012;61(9):2289-2298.

56. Florian S, et al. Loss of GPx2 increases apoptosis, mitosis, and GPx1 expression in the intestine of mice. Free Radic Biol Med. 2010;49(11):1694-1702.

57. Schindelin J, et al. Fiji: an open-source platform for biological-image analysis. Nat Methods. 2012;9(7):676-682.

58. Castro JP, Ott C, Jung T, Grune T, Almeida H. Carbonylation of the cytoskeletal protein actin leads to aggregate formation. Free Radic Biol Med. 2012;53(4):916-925.

59. Klöting N, et al. Insulin-sensitive obesity. Am J Physiol Endocrinol Metab. 2010;299(3):E506-E515. 